\title{
1 A novel and conserved cell wall enzyme that can substitute for the Lipid II \\ 2 synthase MurG
}

3

4 L. Zhang*, K. Ramijan*, V.J. Carrión, L.T van der Aart, J. Willemse, G.P. van Wezel ${ }^{\dagger}$, and

5 D. Claessen ${ }^{\dagger}$.

6

7 Molecular Biotechnology, Institute of Biology, Leiden University, P.O. Box 9505, 2300 RA

8 Leiden, The Netherlands

9

$10 * \quad$ These authors contributed equally

$11 \dagger \quad$ For correspondence: g.wezel@biology.leidenuniv.nl or

$12 \quad$ d.claessen@biology.leidenuniv.nl

13

14

15 Key words: Peptidoglycan, MurG, L-form, morphology switch, cell wall biosynthesis

16 


\section{ABSTRACT}

18 The cell wall is a stress-bearing structure and a unifying trait in bacteria. Without exception,

19 synthesis of the cell wall involves formation of the precursor molecule Lipid II by the activity

20 of the essential biosynthetic enzyme MurG, which is encoded in the division and cell wall

21 synthesis $(d c w)$ gene cluster. Here we present the discovery of a novel cell wall enzyme that

22 can substitute for MurG. A mutant of Kitasatospora viridifaciens lacking a significant part of

23 the $d c w$ cluster including murG surprisingly produced Lipid II and wild-type peptidoglycan.

24 Genomic analysis identified a distant murG paralogue, which encodes a putative enzyme

25 that shares only around $31 \%$ aa sequence identity with MurG. We show that this enzyme

26 can replace the canonical MurG, and we therefore designated it MurG2. Orthologues of

27 murG2 are present in $38 \%$ of all genomes of Kitasatosporae and members of the sister

28 genus Streptomyces. CRISPRi experiments showed that $K$. viridifaciens murG2 can also

29 functionally replace murG in Streptomyces coelicolor, thus validating its bioactivity and

30 demonstrating that it is active in multiple genera. Altogether, these results identify MurG2 as

31 a bona fide Lipid II synthase, thus demonstrating plasticity in cell wall synthesis. 


\section{INTRODUCTION}

35 Bacteria are surrounded by a cell wall, which is a highly dynamic structure that provides

36 cellular protection and dictates cell shape. A major component of the cell wall is

37 peptidoglycan (PG), which is widely conserved in the bacterial domain. Its biosynthesis has

38 been studied for many decades, reinforced by the notion that many successful antibiotics

39 target important steps in this pathway. The first steps of the PG synthesis pathway occur in

40 the cytoplasm, where the peptidoglycan precursor UDP-MurNAc-pentapeptide is

41 synthesized by the consecutive activity of a number of so-called Mur enzymes (MurA-F) ${ }^{1}$.

42 Next, this pentapeptide precursor is linked to undecaprenyl phosphate (or bactoprenol)

43 residing in the plasma membrane by MurX (or MraY), yielding Lipid I. UDP-N-

44 acetylglucosamine--N-acetylmuramyl-(pentapeptide) pyrophosphoryl-undecaprenol N-

45 acetylglucosamine transferase (MurG) then adds the sugar nucleotide UDP-GIcNAc to

46 Lipid I to form Lipid II, which is the complete PG subunit that is flipped to the external side

47 of the membrane. Among the candidates to mediate this flipping, FtsW, MurJ and AmJ have

48 been proposed ${ }^{2-4}$. Following flipping to the exterior of the cell, the PG subunit is then used

49 to synthesize glycan strands by the activity of transglycosylases, after which these strands

50 are cross-linked using transpeptidases ${ }^{5-8}$. Many of the genes required for the biosynthesis

51 of PG and for cell division are located in the so-called dcw gene cluster (for division and cell

52 wall synthesis ${ }^{9,10}$ (see Fig. S1). The content and organization of the dcw cluster are

53 generally conserved among species with similar morphologies, indicating a putative role in

54 bacterial cell shape ${ }^{11}$.

55 Members of the Streptomycetaceae within the Actinobacteria are filamentous Gram-

56 positive soil bacteria that have a complex multicellular life cycle ${ }^{12,13}$. The best-studied genus

57 is Streptomyces, which is industrially highly relevant as it produces over half of all known

58 antibiotics used in the clinic, and many other bioactive compound with clinical or agricultural

59 application ${ }^{14,15}$. The life cycle of streptomycetes starts with the germination of a spore, and 
60 the arising vegetative hyphae grow out via tip extension and branching to form a dense

61 network called the vegetative mycelium. The vegetative mycelium consists of long

62 multinucleated syncytial cells separated by widely spaced crosswalls ${ }^{16,17}$. The reproductive

63 phase is initiated by the formation of an aerial mycelium, whereby the vegetative hyphae are

64 cannibalized as a substrate ${ }^{18,19}$. The aerial hyphae then differentiate into chains of

65 unigenomic spores. During sporulation, the conserved cell division protein FtsZ initially

66 assembles in long filaments in the aerial hyphae, then as regular foci, to finally form a ladder

67 of Z-rings ${ }^{20}$. Eventually, cytokinesis results in spore formation, following a complex process

68 of coordinated cell division and DNA segregation ${ }^{21,22}$.

69 Comparison between Bacillus and Streptomyces shows that some cell division-

70 related proteins have evolved different functionalities between Firmicutes and

71 Actinobacteria. An example of such a divergent function is exemplified by DivIVA: in Bacillus

72 subtilis this protein is involved in selection of the division site by preventing polar

73 accumulation of $\mathrm{FtsZ}^{23}$, while DivIVA in Actinobacteria plays an essential role in polar growth

$74{ }^{24}$. Thus, divIVA cannot be deleted in Actinobacteria while it is dispensable in $B$. subtilis.

75 Conversely, many cell division genes, including ftsZ, can be deleted in Actinobacteria while

76 being essential for unicellular microbes. This makes Actinobacteria intriguing model systems

77 for the study of cell division and growth ${ }^{21,25}$. It is also worth noticing the Streptomycetes

78 have a complex cytoskeleton, with many intermediate filament-like proteins required for

79 hyphal integrity ${ }^{26-29}$.

80 Besides the genus Streptomyces, the family of Streptomycetaceae also

81 encompasses the genera Kitasatospora and Streptacidiphilus. While highly similar in growth

82 and development, Kitasatospora is distinct from Streptomyces ${ }^{30,31}$. We recently described

83 that Kitasatospora viridifaciens releases cell wall-deficient cells, called S-cells, under

84 conditions of hyperosmotic stress ${ }^{32}$. These S-cells are only transiently wall-deficient and

85 can switch to the mycelial mode-of-growth. In some cases, however, prolonged exposure to 
86 high levels of osmolytes can lead to the emergence of mutants that are able to proliferate in

87 the wall-deficient state as so-called L-forms ${ }^{32,33}$. Like S-cells, these L-forms retain the ability

88 to construct functional peptidoglycan based on the observation that removal of the

89 osmolytes from the medium led to the formation of mycelial colonies. L-forms can also be

90 generated in most other bacteria by exposing cells to compounds that target the process of

91 cell wall synthesis ${ }^{33-35}$. Strikingly, such wall-deficient cells that are able to propagate without

92 the FtsZ-based cell division machinery ${ }^{35-37}$. Even though the procedures used to generate

93 L-forms can markedly differ, their mode-of-proliferation is conserved across species and

94 largely based on biophysical principles. An imbalance in the cell surface area to volume ratio

95 in cells that increase in size causes strong deformations of the cell membrane, followed by

96 the release of progeny cells by blebbing, tubulation and vesiculation ${ }^{32,38}$. Given that lipid

97 vesicles without any content are able to proliferate in a similar manner to that observed for

98 L-forms led to the hypothesis that this mode of proliferation may be comparable to that used

99 by early life forms that existed before the cell wall had evolved ${ }^{39,40}$.

$100 \quad$ Here, we exploited the unique properties of a K. viridifaciens L-form strain that readily

101 switches between a wall-deficient and filamentous mode-of-growth to discover a novel

102 MurG-like enzyme that is important for building the PG-based cell wall. Our data surprisingly

103 show that $K$. viridifaciens produces wild-type peptidoglycan in the absence of murG, which

104 was so far considered essential for Lipid II biosynthesis in all bacteria. The MurG activity is

105 taken over by a novel paralogue called MurG2, which occurs widespread in filamentous

106 actinobacteria, and able to substitute for the absence of MurG across different genera.

\section{RESULTS}

109 Morphological transitions of the shape-shifting strain alpha

110 We recently generated a $K$. viridifaciens L-form lineage by exposing the parental wild-type

111 strain to high levels of penicillin and lysozyme. This strain, designated alpha, proliferates 
112 indefinitely in the cell wall-deficient state in media containing high levels of osmolytes ${ }^{32}$

113 (Table S1). On solid LPMA medium, alpha forms green-pigmented viscous colonies, which

114 exclusively contain L-form cells (Fig. 1A). In contrast, the parental strain forms compact and

115 yellowish colonies composed of mycelia and S-cells on LPMA medium (Fig. 1B). Likewise,

116 in liquid LPB medium alpha exclusively proliferates in the wall-deficient state, in a manner

117 that is morphologically similar to that described for other L-forms ${ }^{35,41,42}$; (Extended Data

118 Video S1; Fig. 1C). Following strong deformations of the mother cell membrane (see panels

119 of 56, 150, and 200 min in Fig. 1C), small progeny cells are released after approximately

$120300 \mathrm{~min}$. The mother cell, from which the progeny was released (indicated with an asterisk)

121 lysed after 580 min. Characterization using transmission electron microscopy (TEM)

122 confirmed that alpha possessed no PG-based cell wall when grown on media containing

123 high levels of osmolytes (Fig. 1D). Notably, when alpha is plated on MYM medium (lacking

124 high levels of osmolytes) the strain can switch to the mycelial mode-of-growth (Fig. 1E).

125 However, unlike the wild-type strain (Fig. 1F), the mycelial colonies of alpha fail to develop

126 aerial hyphae and spores. Subsequent transfer of mycelia to LPMA plates stopped

127 filamentous growth and reinitiated wall-deficient growth, during which L-form cells are

128 extruded from stalled hyphal tips (Extended Data Video S2; Fig. 1G). Given the ability of

129 these wall-deficient cells to proliferate, they eventually dominated the culture (not shown).

130 Taken together, these results demonstrate that alpha can switch between a walled and wall-

131 deficient state.

133 Deletion of divIVA abolishes switching of alpha from the wall-deficient to the

134 filamentous mode-of-growth

135 The ability of alpha to efficiently switch between the walled and wall-deficient state provides

136 an ideal platform to delete genes essential for cell wall biosynthesis. As a proof-of-concept,

137 we focused on $\operatorname{divIVA}$, which is essential for polar growth in filamentous actinomycetes ${ }^{24}$. 
138 In Actinobacteria, divIVA is located adjacent to the conserved dcw gene cluster (Fig. S1).

139 divIVA is present in Gram-positive rod-shaped (Mycobacterium, Corynebacterium, Bacillus),

140 filamentous (Streptomyces and Kitasatospora) and coccoid (Staphylococcus and

141 Streptococcus) bacteria, but absent in Gram-negatives such as Escherichia coli. In $B$.

142 subtilis and Staphylococcus aureus, the DivIVA proteins share only 29\% (BSU15420) and

$14326 \%$ (SAOUHSC_01158) aa identity to the S. coelicolor orthologue. To localize DivIVA,

144 plasmid pKR2 was created, allowing constitutive expression of DivIVA-eGFP (Table S2).

145 Fluorescence microscopy revealed that the fusion protein localized to hyphal tips (Fig. S2A),

146 similarly as in streptomycetes ${ }^{24}$. When alpha was grown in the wall-deficient state in LPB

147 medium, typically one or two foci of DivIVA-eGFP were detected per cell, which invariably

148 were localized to the membrane. In contrast, no foci were detected in L-form cells containing

149 the empty plasmid (pKR1) or those expressing cytosolic eGFP (pGreen ${ }^{43}$ ). We then

150 constructed the plasmids pKR3 to delete divIVA and pKR4 to delete a large part of the dcw

151 gene cluster, including divIVA (Table S2). Introduction of these plasmids into alpha by PEG-

152 mediated transformation and a subsequent screening yielded the desired $\operatorname{divIVA}$ and $d c w$

153 mutants (Fig. S3). Analysis of growth in LPB medium or on solid LPMA plates indicated that

154 the L-form cells proliferated normally in the absence of divIVA or part of the dcw gene cluster

155 (Fig. 2). However, when L-form cells were plated on MYM medium (lacking

156 osmoprotectants), only the alpha strain was able to switch to the mycelial mode-of-growth

157 (Fig. 2B). Introduction of plasmid pKR6, which expresses divIVA from the constitutive gap1

158 promoter, complemented growth of the divIVA mutant on MYM medium (Fig. 2B). In

159 agreement, Western blot analysis using antibodies against DivIVA of Corynebacterium

160 glutamicum confirmed the absence of DivIVA in both the divIVA and the dcw mutant, and

161 also showed the expression was restored in the divIVA mutant complemented with pKR6

162 (Fig. 2C).

163 To analyse if the switch from the wall-deficient to the walled state in the absence of 
164 DivIVA was blocked due to the failure to produce the cytosolic precursors required for

165 peptidoglycan synthesis in the L-form state, we performed a comparative LC-MS analysis

166 (Fig. 2D). We noticed that the LC-MS profiles of the divIVA and $d c w$ mutant strains were

167 similar to that of alpha with respect to the cytosolic PG building blocks (Fig. 2D). Importantly,

168 MS-MS analysis identified the last cytosolic precursor in the PG biosynthesis pathway, UDP-

169 MurNAc-pentapeptide $(\mathrm{Mw}=1194.35)$ in all strains (Fig. 2E). Taken together, these results

170 demonstrate that DivIVA is essential for filamentous growth but not required for synthesis of

171 the cytosolic PG precursors.

172

173 Identification of a distant MurG paralogue as a novel Lipid II synthase

174 Having a mutant lacking many genes of the dcw cluster offers many opportunities for the 175 study of individual genes. The constructed $d c w$ mutant lacks ftsW, murG, ftsQ, ftsZ, ylmD, $176 y \operatorname{lmE}, \operatorname{sep} F, \operatorname{sep} G$, and $\operatorname{divIVA}$. Surprisingly, introduction of only divIVA (expressed from the 177 constitutive gap1 promoter) restored the ability of the dcw mutant to switch to the walled 178 mode-of-growth on solid media lacking osmoprotectants (Fig. 3). The colonies that were 179 formed, were small and heterogeneous as compared to the mycelial colonies formed by 180 alpha (Fig. 3A). Furthermore, expression of divIVA in the dcw mutant was not able to restore

181 filamentous growth in liquid cultures (data not shown). To verify that the dcw mutant 182 expressing divIVA produced normal PG on solid medium, we performed a peptidoglycan 183 architecture analysis using LC-MS (Fig. 3B). This surprisingly revealed that all expected 184 muropeptides were formed at levels comparable to those formed by alpha and the wild-type 185 strain, despite the absence of a functional murG (Fig. 3B; Table 1).

186 The ability of the dcw mutant expressing divIVA to grow filamentous inevitably means 187 that another protein had functionally replaced the activity of MurG. Blast analysis of the 188 amino acid sequence of MurGsco (SCO2084) against the genome sequence of $K$. 189 viridifaciens revealed that this actinomycete contains two putative, but distant MurG 
190 homologs (Supplemefntary Table 4). The two additional homologs (BOQ63_RS12640 and

191 BOQ63_RS05415) showed 31.2\% and 16.5\% sequence identity, respectively, to MurG

192 (BOQ63_RS32465). Further investigation revealed that MurG proteins possess two

193 characteristic domains: an N-terminal domain that contains the Lipid I binding site (PF03033)

$194{ }^{44}$, and a C-terminal domain that contains the UDP-GlcNAc binding site (PF04101; Fig. S4),

195 both of which are required for the UDP-N-acetyl-glucosamine transferase activity. Of the two

196 distant MurG homologues, only BOQ63_RS12640 contained both domains (Fig. S4). A

197 broader search of MurG-like proteins in other Streptomyces and Kitasatospora spp. revealed

198 that $38 \%$ of the strains possess one, two and sometimes even three genes for MurG-like

199 proteins containing both the necessary N-terminal (PF03033) and C-terminal (PF04101)

200 domains (Fig. 4), in addition to the canonical MurG, which is present in all strains and

201 encoded in the $d c w$ gene cluster. A sequence similarity network was produced by pairwise

202 comparing the 1553 MurG and MurG-like proteins extracted from all translated

203 Streptomyces and Kitasatospora genomes, which showed that nearly all MurG proteins

204 encoded by the orthologue of murG in the $d c w$ gene cluster grouped together. However, the

205 MurG-like proteins clustered in many different groups (Fig. S5).

206 To corroborate that murG is not required for filamentous growth, we decided to delete

207 murG in alpha using knock-out construct pKR8 (Table S2). The genotype of the mutant was

208 verified by PCR (Fig. S6) and showed that the absence of murG had no effect on L-form or

209 filamentous growth (Fig. 5). Likewise, inactivation of murG2 in alpha using construct pKR9

210 had no effect on L-form growth and did not prevent switching to mycelial growth (Fig. 5). We

211 then attempted to create a double mutant by deleting murG2 in the murG mutant. PCR

212 analysis on a putative double mutant strain with the highly sensitive Q5 DNA polymerase

213 indicated, however, that a small proportion of the multinucleated L-forms had retained a

214 copy of murG2 (Fig. S6). Also, further subculturing of this merodiploid strain in the presence

215 of antibiotics that counter-selected for maintenance of murG2 did not lead to a complete loss 
216 of this gene, suggesting that the ability to produce Lipid II is essential in these L-forms (see

217 Discussion). Nevertheless, plating this merodiploid strain on MYM medium essentially

218 blocked mycelial growth, and only at very high cell densities infrequent shifters were found

219 (see encircled colony in Fig. 5A).

220 Having demonstrated that murG is not required for filamentous growth of alpha, we

221 then wondered whether murG would also be dispensable for filamentous growth of the wild-

222 type strain. Notably, murG deletion mutants could not be obtained if transformants were

223 selected on MYM medium, unlike a murG2 deletion mutant that was readily found. However,

224 when transformants were selected on LPMA medium containing high levels of sucrose, a

225 murG mutant could be created in K. viridifaciens (see Fig. S7). As shown in Figure 5B, the

226 generated murG and murG2 mutants were able to develop and sporulate normally on MYM,

227 when compared to the parental wild type. However, exposing the strains to low levels of

228 penicillin and ampicillin revealed that the murG mutant was more susceptible to these cell

229 wall-targeting antibiotics when compared to the wild-type and its murG2 mutant. By contrast,

230 no difference effect was observed when tetracycline was added to the plates (Fig. 5C).

231 Altogether, these results demonstrate that MurG and MurG2 have overlapping activities,

232 whereby MurG2 is able to functionally replace the canonical Lipid II synthase MurG.

234 MurG2 from K. viridifaciens can functionally replace MurG in S. coelicolor

235 The observations that murG2 can functionally replace murG in $K$. viridifaciens and that

236 strains expressing only MurG2 produce wild-type peptidoglycan, strongly suggest that the

237 murG2 gene product synthesizes Lipid II. To further substantiate this, we investigated

238 whether murG2 could also functionally complement murG (SCO2084) in another

239 Actinobacterium, namely the model organism Streptomyces coelicolor M145, which itself

240 does not harbour an orthologue of murG2. For this, we created construct pGWS1379

241 expressing murG2 from the constitutive ermE promoter in the integrative vector pMS82 and 
242 introduced it into S. coelicolor. As a control we used the empty vector pMS82. We then

243 applied CRISPRi ${ }^{45}$ to knock-down the native murG to assess viability. CRISPRi only works

244 when the spacer of the dCas9/sgRNA complex targets the non-template strand of murGsco,

245 and not the template strand, or when the spacer is absent ${ }^{45,46}$. The functionality of the

246 CRISPRi constructs was evident in control cells without murG2; colonies expressing the

247 dCas9/sgRNA complex targeting the non-template strand of murGsc in M145 could hardly

248 grow, due to the essential function of murG. Conversely, control transformants harboring

249 CRISPRi constructs targeting the template strand or without spacer (empty plasmid) grew

250 normally (Fig. 6). Excitingly, S. coelicolor transformants expressing murG2 grew apparently

251 normally under all conditions, even when murG expression was knocked down by the

252 CRISPRi system. This validates the concept that murG2 can functionally replace the

253 canonical murG (Fig. 6). Taken together, our experiments show that the MurG2 enzyme can

254 functionally replace the Lipid II-biosynthetic enzyme MurG, both in Kitasatospora and in

255 Streptomyces.

\section{DISCUSSION}

258 The cell wall is a hallmark feature of bacterial cells, and the steps involved in its biosynthesis

259 are widely conserved across the bacterial domain. In all bacteria, the final cytosolic step in

260 precursor biosynthesis is the conversion of Lipid I to Lipid II by MurG encoded in the dcw

261 gene cluster. We here show for the first time that the novel enzyme MurG2 can replace the

262 activity of MurG and demonstrate that murG is dispensable in the filamentous actinomycete

263 K. viridifaciens in the presence of murG2. MurG2 alone is sufficient to produce wild-type

264 peptidoglycan. MurG2 is in fact widespread among the Streptomycetaceae and was

265 identified in the genomes of $38 \%$ of all Streptomyces and Kitasatospora strains.

266 Furthermore, introduction of K. viridifaciens murG2 into S. coelicolor M145 - which itself

267 lacks an orthologue of murG2 - allowed the knock-down of the canonical murG using 
268 CRISPRi, showing that the gene is a bona fide cell wall biosynthetic gene that is functional

269 in different Actinobacteria.

270 Filamentous actinomycetes are multicellular bacteria that form networks of

271 interconnected hyphae, whereby sporulating aerial hyphae are established after a period of

272 vegetative growth. Streptomyces is a wonderful model system for the study of cell division,

273 among others because cell division is not required for normal growth of this bacterium ${ }^{21,25,47}$.

274 Most of the cell division proteins are encoded by genes located in the conserved dcw gene

275 cluster. In streptomycetes, many cell division genes such as ftsl, ftsL, ftsW and divlC are

276 only required for sporulation and do not affect normal growth ${ }^{48-50}$. Our data surprisingly show

277 that many genes within the $d c w$ cluster can be deleted simultaneously in $K$. viridifaciens,

278 including divIVA that is essential for polar growth in Actinobacteria, by using a strain (alpha)

279 with the ability to readily switch between a wall-deficient and filamentous mode-of-growth.

280 The alpha strain thus provides a unique system for the identification of proteins that are

281 required for polar growth. As a proof-of-concept for this principle, divIVA that is required for

282 polar growth, was successfully deleted. Consistent with its role in driving apical growth, the

283 absence of divIVA arrested growth in the wall-deficient state but had no effect on synthesis

284 of the PG building blocks. This indicates that the block in PG formation occurred in a later

285 step of the PG biosynthesis pathway. Introduction of only divIVA in the dcw mutant retored

286 polar growth, which was a rather surprising discovery given the absence of a whole string

287 of genes involved in cell division and cell wall synthesis, and in particular murG. MurG

288 catalyzes the coupling of GIcNAc to Lipid I, yielding the PG precursor Lipid II and this

289 enzymatic activity is therefore essential for cell wall synthesis. The ability of alpha to produce

290 a cell wall with an apparently normal architecture, as shown by the analysis of the

291 peptidoglycan, indicated that $K$. viridifaciens possesses other enzymes capable of

292 synthesizing Lipid II in the absence of murG. An in-silico search in the genome of $K$.

293 viridifaciens identified murG2 (BOQ63_RS12640), which is a distant relative of MurG with 
294 the likely ability to replace the activity of the canonical MurG. This is based among others

295 on the presence of the two domains that are known to be required for the transfer of GlcNAc

296 to Lipid I. Many Actinobacteria possess proteins carrying these two domains, suggesting

297 that MurG2 proteins are common in these bacteria. In fact, some species even contain three

298 genes for MurG-like proteins, in addition to the canonical MurG encoded in the dcw gene

299 cluster. Interestingly, murG and murG2 could both be individually deleted in the wild-type

300 strain, whereby the resulting mutants showed normal growth and development when strains

301 were grown in non-stressed environments. However, the murG mutant was more

302 susceptible to cell wall-targeting antibiotics than the wild-type strain or its murG2 mutant.

303 Considering that MurG2 alone suffices to produce normal peptidoglycan, this suggests that

304 MurG is required to build a more robust cell wall. Deletion of murG was only possible after

305 exposing transformants to hyperosmotic growth conditions. We hypothesize that the

306 hyperosmotic conditions activated the transcription of murG2, thus allowing deletion of murG

307 specifically under these growth conditions. This implies that the function of murG2 is to

308 synthesize Lipid II under specific growth conditions, e.g. during hyperosmotic stress.

309 In further support of the function of MurG2 as an alternative Lipid II synthase, we

310 tested if it could also take over the function of murG in another bacterium. For this, we chose

311 the model streptomycete $S$. coelicolor M145, which is a distinct genus within the

312 Streptomycetaceae ${ }^{31,51}$, but lacking a copy of murG2. Importantly, murG could be readily

313 depleted using CRISPRi in strains expressing murG2 from a constitutive promoter, while

314 knock-down of murG in colonies of S. coelicolor harboring control plasmids led to very

315 severe growth defects. This not only validates our data that murG2 encodes a Lipid II

316 synthase, but also that this is a more universal phenomenon that does not only occur in

317 specific strains of Kitasatospora or connect to strains that have the capacity to produce

318 natural wall-less cells. Furthermore, it shows that no additional Kitasatospora genes are

319 required to allow murG2 to functionally complement murG in Streptomyces. 
We also attempted to delete murG and murG2 simultaneously in alpha. While the

321 single mutants were readily obtained, we never obtained strains that completely devoid of

322 both murG and murG2, despite many attempts. Like mycelia, L-forms are multinucleated

323 cells, and some cells of the population retained murG2, most likely to ensure minimal levels

324 of Lipid II. Consistent with this idea is the finding that antibiotics that target Lipid II, such as

325 vancomycin, are lethal to alpha (our unpublished data). We hypothesize that this lethality is

326 caused by depletion of the lipid carrier undecaprenyl diphosphate, which is also used in

327 other pathways and which may be essential for these L-forms. Removing murG2 in strains

328 lacking murG strain virtually blocked the ability to switch to the filamentous mode-of-growth,

329 whereas each of the single mutants switched as efficiently as the parental alpha strain. Thus,

330 we show that MurG2 is a novel enzyme involved in cell wall metabolism, which appears to

331 facilitate switching between a wall-deficient and a walled lifestyle.

333 MATERIALS AND METHODS

\section{Strains and media}

335 Bacterial strains used in this study are shown in Table S1. To obtain sporulating cultures of

$336 \mathrm{~K}$. viridifaciens and S. coelicolor, strains were grown at $30^{\circ} \mathrm{C}$ for 4 days on MYM medium ${ }^{52}$.

337 For general cloning purposes, E. coli strains DH5a and JM109 were used, while E. coli

338 ET12567 and SCS110 were used to obtain unmethylated DNA. E. coli strains were grown

339 at $37^{\circ} \mathrm{C}$ in LB medium, supplemented with chloramphenicol $\left(25 \mu \mathrm{g} \mathrm{ml}^{-1}\right)$, ampicillin $(100 \mu \mathrm{g}$

$\left.340 \mathrm{ml}^{-1}\right)$, apramycin $\left(50 \mu \mathrm{g} \mathrm{ml} l^{-1}\right)$, kanamycin $\left(50 \mu \mathrm{g} \mathrm{ml}^{-1}\right)$, or viomycin $\left(30 \mu \mathrm{g} \mathrm{ml}^{-1}\right)$, where

341 necessary.

342 To support growth of wall-deficient cells, strains were grown in liquid LPB medium

343 while shaking at $100 \mathrm{rpm}$, or on solid LPMA medium at $30^{\circ} \mathrm{C}^{32}$. To switch from the wall-

344 deficient to the filamentous mode-of-growth, L-form colonies grown on LPMA for seven days

345 were streaked on MYM medium. If needed, mycelial colonies of switched strains were 
346 transferred after 4 days to liquid TSBS medium and grown for two days at $30^{\circ} \mathrm{C}$, while

347 shaking at $200 \mathrm{rpm}$.

\section{Construction of plasmids}

350 All plasmids and primers used in this work are shown in Tables S2 and S3, respectively.

352 Construction of the DivIVA localization construct pKR2

353 To localize DivIVA, we first created plasmid pKR1 containing a viomycin resistance

354 cassette cloned into the unique Nhel site of plJ8630 ${ }^{53}$. To this end, the viomycin resistance

355 cassette was amplified from plJ780 ${ }^{54}$ with the primers $v p h-F W-N h e l$ and vph-RV-Nhel.

356 Next, we amplified the constitutive gap1 promoter as a 450 bp fragment from the genome

357 of S. coelicolor with the primers Pgap1-FW-Bglll and Pgap1-RV-Xbal. We also amplified the

358 divIVA coding sequence (the +1 to +1335 region relative to the start codon of $\operatorname{divIVA}$

359 (BOQ63_RS32500) from the chromosome of $K$. viridifaciens using primers divIVA-FW-Xbal

360 and divIVA-Nostop-RV-Ndel ${ }^{55}$. Finally, the promoter and $\operatorname{divIVA}$ coding sequence were

361 cloned into pKR1 as a Bglll/Xbal and Xbal/Ndel fragment respectively, yielding plasmid 362 pKR2.

364 Construction of the deletion constructs $p K R 3, p K R 4, p K R 8, p K R 9$ and $p K R 10$

365 The divIVA mutant was created in $K$. viridifaciens using pKR3, which is a derivative of the 366 unstable plasmid pWHM3 ${ }^{56}$. In the divIVA mutant, nucleotides +205 to +349 relative to the 367 start codon of diviVA were replaced with the loxP-apra resistance cassette as described ${ }^{57}$. 368 A similar strategy was used for the deletion of the partial $d c w$ cluster (plasmid pKR4), and

369 for the deletion of murG (plasmid pKR8) and murG2 (plasmid pKR9). For the deletion of the 370 partial $d c w$ cluster, the chromosomal region from +487 bp relative to the start of the ftsW 371 gene (BOQ63_RS32460) until +349 relative to the start of the divIVA gene were replaced 
372 with the apramycin resistance marker. For the deletion of murG (BOQ63_RS32465, located

373 in the $d c w$ cluster), the nucleotides +10 to +1077 bp relative to the start codon of murG were

374 replaced with the loxP-apra resistance cassette, while for the murG2 (BOQ63_RS12640)

375 deletion the chromosomal region from +18 to +1105 bp relative to the start of murG 2 were

376 replaced with the apramycin resistance marker. To construct the murG/murG2 double

377 mutant, pKR10 was created, replacing the apramycin resistance cassette in pKR8 by a

378 viomycin resistance cassette. To this end, the viomycin resistance cassette was amplified

379 from plJ780 ${ }^{54}$ with the primers $v p h-F w-E c o R I-H i n d I I-X b a l$ and vph-Rv-EcoRI-HindIII-Xbal.

380 The viomycin resistance cassette contained on the PCR fragment was then cloned into

381 pKR8 using Xbal, thereby replacing the apramycin cassette and yielding pKR10.

382

383 Construction of the complementation constructs $p K R 6$ and $p K R 7$

384 For complementation of divIVA under control of the strong gap 1 promoter ${ }^{43}$, the constructs

385 pKR6 was made. First, we created plasmid pKR5 with the strong gap1 promoter. The

386 promoter region of gap1 (SCO1947) was amplified with the primers Pgap1-FW-Bglll and

387 Pgap1-RV-Xbal using S. coelicolor genomic DNA as the template. Next, the gap1 promoter

388 was cloned as Bglll/Xbal fragment into the integrative vector plJ8600 ${ }^{53}$ to generate the

389 plasmid pKR5. Afterwards, the divIVA coding sequence was amplified from the genome of

390 K. viridifaciens with the primers divIVA-Xbal-FW and divIVA-Ndel-RV. Finally, to create the

391 plasmid pKR6 the Xbal/Ndel fragment containing the divIVA coding sequence was cloned

392 in pKR5.

393

394 Construction of the murG2 expression construct pGWS1379

395 A DNA fragment containing the $\mathrm{ermE}^{*}$ promoter was obtained as an EcoRI-Ndel fragment

396 from pHM10a ${ }^{58}$, while murG2 was amplified by PCR from $K$. viridifaciens chromosomal DNA

397 using primer pair murG2_F+4_ENdel and murG2_R+1146_HX. The ermEp* fragment and 
398 Ndel-Xbal-digested murG2 were simultaneously cloned into EcoRI-Xbal digested pSET152

399 to generate construct pGWS1378. The insert of pGWS1378 was then introduced as a Pvull

400 fragment into EcoRV-digested pMS82 ${ }^{59}$ to generate construct pGWS1379. This construct

401 was then introduced into S. coelicolor M145 via protoplast transformation as described ${ }^{60}$.

\section{Transformation of L-forms}

404 Transformation of alpha essentially followed the protocol for the rapid small-scale

405 transformation of Streptomyces protoplasts ${ }^{60}$, with the difference that $50 \mu \mathrm{l}$ cells from a mid406 exponential growing L-form culture were used instead of protoplasts. Typically, $1 \mu$ DNA

407 was used for each transformation. Transformants were selected by applying an overlay 408 containing the required antibiotics in P-buffer after 20 hours. Further selection of

409 transformants was done on LPMA medium supplemented with apramycin $\left(50 \mu \mathrm{g} \mathrm{ml}{ }^{-1}\right)$,

410 thiostrepton $\left(5 \mu \mathrm{g} \mathrm{ml}^{-1}\right)$, or viomycin $\left(30 \mu \mathrm{g} \mathrm{ml}^{-1}\right)$, when necessary. Transformants were

411 verified by PCR (Table S3).

\section{3 murGsco (SCO2084) knockdown via CRISPRi}

414 The Ncol restriction site within the integrase gene of phage $\varphi$ C31 in pSET152 was removed

415 by introducing a silent GCC to GCG change in codon A360 via site-directed mutagenesis by

416 PCR using primer pairs 152DNcol_F and 152DNcol_R, to generate construct pGWS1369.

417 Subsequently, a DNA fragment containing the sgRNA scaffold (no spacer) and Pgapdh-

418 dcas9 of constructs pGWS1049 ${ }^{46}$ was cloned as an EcoRI-Xbal fragment into pGWS1369

419 to generate construct pGWS1370. The $20 \mathrm{nt}$ spacer sequence was introduced into sgRNA

420 scaffold by PCR using forward primers SCO2084_T_F or SCO2084_NT5_F together with

421 the reverse primer SgTermi_R_B. The PCR products were cloned as Ncol-BamHI fragments

422 into pGWS1370 to generate constructs pGWS1371 (targeting the template strand of 423 SCO2084) and pGWS1376 (targeting the non-template strand of SCO2084). Constructs 
424 pGWS1370 (no spacer), pGWS1371 (targeting the template strand) and pGWS1376

425 (targeting the non-template strand) were introduced into S. coelicolor M145+pMS82 (empty

426 plasmid) and M145+pGWS1379 (expressing murG2) via protoplast transformation as

427 described previously ${ }^{60}$.

\section{Microscopy}

430 Strains grown in LPB or LPMA were imaged using a Zeiss Axio Lab A1 upright microscope

431 equipped with an Axiocam Mrc. A thin layer of LPMA (without horse serum) was applied to

432 the glass slides to immobilize the cells prior to the microscopic analysis.

434 Fluorescence microscopy

435 Fluorescence microscopy pictures were obtained with a Zeiss Axioscope A1 upright

436 fluorescence microscope equipped with an Axiocam Mrc5 camera. Aliquots of $10 \mu$ of live

437 cells were immobilized on top of a thin layer of LPMA (without horse serum) prior to analysis.

438 Fluorescent images were obtained using a 470/40 nm band pass excitation and a 505/560

439 band pass detection, using an 100x N.A. 1.3 objective. To obtain a sufficiently dark

440 background, the background of the images was set to black. These corrections were made

441 using Adobe Photoshop CS5.

\section{Time-lapse microscopy}

444 To visualize the proliferation of alpha, cells were collected and resuspended in $300 \mu \mathrm{LPB}$

445 (containing 4-22\% sucrose) and placed in the wells of a chambered 8-well $\mu$-slide (ibidiß).

446 Cells were imaged on a Nikon Eclipse Ti-E inverted microscope equipped with a confocal

447 spinning disk unit (CSU-X1) operated at 10,000 rpm (Yokogawa), using a 40x Plan Fluor

448 Lens (Nikon) and illuminated in bright-field. Images were captured every 2 minutes for 10-

44915 hours by an Andor iXon Ultra 897 High Speed EM-CCD camera (Andor Technology). Z- 
450 stacks were acquired at 0.2-0.5 $\mu \mathrm{m}$ intervals using a NI-DAQ controlled Piezo element.

451 During imaging wall-less cells were kept at $30^{\circ} \mathrm{C}$ using an INUG2E-TIZ stage top incubator

452 (Tokai Hit).

453

454 Electron microscopy

455 For transmission electron microscopy, L-forms obtained from a 7-day-old liquid-grown alpha

456 culture were trapped in agarose blocks prior to fixation with $1.5 \%$ glutaraldehyde and a post-

457 fixation step with $1 \%$ OsO4. Samples were embedded in Epon and sectioned into $70 \mathrm{~nm}$

458 slices. Samples were stained using uranyl-acetate $(2 \%)$ and lead-citrate $(0.4 \%)$, if

459 necessary, before being imaged using a Jeol 1010 or a Fei 12 BioTwin transmission electron

460 microscope.

461

462 DivIVA detection using Western analysis

463 To detect DivIVA using Western analysis, the biomass of L-form strains was harvested after

4647 days of growth in LPB medium, while biomass of mycelial strains was obtained from liquid-

465 grown TSBS cultures after 17 hours. Cell pellets were washed twice with 10\% PBS, after

466 which they were resuspended in 50 mM HEPES pH 7.4, $50 \mathrm{mM} \mathrm{NaCl}, 0.5 \%$ Triton X-100, 1

467 mM PFMS and P8465 protease inhibitor cocktail (Sigma). The cells and mycelia were

468 disrupted with a Bioruptor Plus Sonication Device (Diagenode). Complete lysis was verified

469 by microscopy, after which the soluble cell lysate was separated from the insoluble debris

470 by centrifugation at $13,000 \mathrm{rpm}$ for $10 \mathrm{~min}$ at $4^{\circ} \mathrm{C}$. The total protein concentration in the cell

471 lysates was quantified by a BCA assay (Sigma-Aldrich). Equal amounts of total proteins

472 were separated with SDS-PAGE using $12,5 \%$ gels. Proteins were transferred to

473 polyvinylidene difluoride (PVDF) membranes (GE Healthcare) with the Mini Trans-Blot@ Cell

474 (Bio-Rad Laboratories) according to the manufacturer's instructions. DivIVA was detected

475 using a 1:5,000 dilution of polyclonal antibodies raised against Corynebacterium glutamicum 
476 DivIVA (kindly provided by Professor Marc Bramkamp). The secondary antibody, anti-rabbit

477 IgG conjugated to alkaline phosphatase (Sigma), was visualized with the BCIP/NBT Color

478 Development Substrate (Promega).

\section{Isolation of cytoplasmic peptidoglycan precursors}

481 For the cytoplasmic PG precursor isolation and identification, we used a modification of the

482 method previously described ${ }^{61}$. The alpha strain and the divIVA and dcw mutants were

483 grown in LPB for seven days, while the wild-type $K$. viridifaciens strain was grown for three

484 days in a modified version of LPB lacking sucrose. The cells were harvested by

485 centrifugation at $4^{\circ} \mathrm{C}$ and washed in $0,9 \% \mathrm{NaCl}$. Cells were extracted with $5 \%$ cold trichloric

486 acid (TCA) for 30 minutes at $4^{\circ} \mathrm{C}$. The extracts were centrifuged at $13,000 \mathrm{rpm}$ for 5 minutes

487 at $4^{\circ} \mathrm{C}$, after which the supernatants were desalted on a Sephadex G-25 column (Illustra

488 NAP-10 Columns, GE Healthcare, Pittsburgh) and concentrated by rotary evaporation. The

489 concentrated precursors were dissolved in $200 \mu$ I HPLC-grade water.

\section{Peptidoglycan extraction}

492 The peptidoglycan architecture was analyzed as described ${ }^{62}$. Mycelia of the wild-type strain,

493 alpha and the dcw mutant complemented with divIVA were grown on top of cellophane discs

494 on modified LPMA medium lacking sucrose and horse serum. Following growth, the mycelial

495 mass was removed from the cellophane, washed in $0.1 \mathrm{M}$ Tris- $\mathrm{HCl} \mathrm{pH} 7.5$ and lyophilized.

$49610 \mathrm{mg}$ of the lyophilized biomass was used for PG isolation. Therefore, the biomass was

497 boiled in $0.25 \%$ SDS in $0.1 \mathrm{M} \mathrm{Tris} / \mathrm{HCl} \mathrm{pH} 6.8$, thoroughly washed, sonicated, and treated

498 with DNase, RNase and trypsin. Inactivation of these enzymes was performed by boiling the

499 samples followed by washing with water. Wall teichoic acids were removed with $1 \mathrm{M} \mathrm{HCl}^{63}$.

500 PG was digested with mutanolysin and lysozyme. Muropeptides were reduced with sodium

501 borohydride and the $\mathrm{pH}$ was adjusted to 3.5-4.5 with phosphoric acid. 


\section{$502 \quad$ LC-MS analysis of PG precursors and muropeptides}

503 The LC-MS setup consisted of a Waters Acquity UPLC system (Waters, Milford, MA, USA)

504 and an LTQ Orbitrap XL Hybrid Ion Trap-Orbitrap Mass Spectrometer (Thermo Fisher

505 Scientific, Waltham, MA, USA) equipped with an Ion Max electrospray source.

506 Chromatographic separation of muropeptides and precursors was performed on an Acquity

507 UPLC HSS T3 C18 column $(1.8 \mu \mathrm{m}, 100 \AA, 2.1 \times 100 \mathrm{~mm})$. Mobile phase A consisted of

$50899.9 \% \mathrm{H}_{2} \mathrm{O}$ and $0,1 \%$ formic acid, while mobile phase $\mathrm{B}$ consisted of $95 \%$ acetonitrile, $4.9 \%$

$509 \mathrm{H}_{2} \mathrm{O}$ and $0,1 \%$ formic acid. All solvents used were of LC-MS grade or better. The flow rate

510 was set to $0.5 \mathrm{ml} \mathrm{min}^{-1}$. The binary gradient program consisted of $1 \mathrm{~min} 98 \% \mathrm{~A}, 12 \mathrm{~min}$ from

$51198 \% \mathrm{~A}$ to $85 \% \mathrm{~A}$, and 2 min from $85 \% \mathrm{~A}$ to $0 \% \mathrm{~A}$. The column was then flushed for $3 \mathrm{~min}$

512 with $100 \% \mathrm{~B}$, after which the gradient was set to $98 \%$ and the column was equilibrated for

$5138 \mathrm{~min}$. The column temperature was set to $30^{\circ} \mathrm{C}$ and the injection volume used was $5 \mu \mathrm{L}$.

514 The temperature of the autosampler tray was set to $8^{\circ} \mathrm{C}$. Data was collected in the positive

515 ESI mode with a scan range of $\mathrm{m} / \mathrm{z} 500-2500$ in high range mode. The resolution was set

516 to 15.000 (at $\mathrm{m} / \mathrm{z} 400)$.

518 Sequence homology analysis of dcw gene clusters

519 The homology search of the different $d c w$ clusters was done using MultiGeneBlast ${ }^{64}$. The

520 query used for the search was the dcw cluster from Streptomyces coelicolor A3(2), for which

521 the required sequences were obtained from the Streptomyces Annotation Sever (StrepDB).

522 The homology search included the loci from SCO2077 (divIVA) until SCO2091 (ftsL). A

523 database was constructed with genome assemblies obtained from NCBI. The analyzed

524 species have the following accession numbers: NC_003888 (S. coelicolor A3(2),

525 NZ_MPLE00000000.1 (Kitasatospora viridifaciens DSM40239), CP000480 (Mycobacterium

526 smegmatis MC2 155), AL123456 (Mycobacterium tuberculosis H37Rv), CP014279

527 (Corynebacterium stationis ATCC 6872), BX927147 (Corynebacterium glutamicum 
528 ATCC13032), AL009126 (Bacillus subtilis subsp.168), U00096 (Escherichia coli K-12),

529 CP000253.1 (Staphylococcus aureus NTC8325), and AE007317 (Streptococcus

530 pneumoniae R6). In the homology search, the Blast parameters were set to a minimal

531 sequence coverage of $25 \%$ and a minimal identity of $30 \%$. The first 11 hits of the

532 MultiGeneBlast output are shown in Fig. S1, where homologs genes are represented by

533 arrows with the same colors.

534

535 Phylogeny analysis of Streptomyces and Kitasatospora species

536 A set of 1050 Streptomyces and Kitasatospora genomes was downloaded from NCBI by

537 querying the fasta files in combination with the taxonomic identifier. To this set, 116

538 unpublished draft genome sequences of an in-house collection of actinomycetes were

539 added ${ }^{65}$. Complete protein sets encoded within the genomes of Streptomyces and

540 Kitasatospora spp. were extracted. The Pfam domains of four housekeeping proteins, AtpD

541 (ATP synthase subunit beta), RecA (recombinase A), TrpB (tryptophan synthase beta chain)

542 and GyrB (DNA gyrase subunit B), were retrieved from https://pfam.xfam.org/ and are

543 annotated as PF00213, PF00154, PF06233 and PF00204, respectively. Using the selected

544 Pfam domains, the HMMsearch program of the HMMER v3.0 package ${ }^{66}$ was employed to

545 identify analogous proteins within the chosen species. MAFFT was used to perform a

546 multiple sequence alignment ${ }^{67}$. Aligned sequences were concatenated using SeqKit ${ }^{68}$ and

547 maximum likelihood phylogenetic trees were calculated with RAxML ${ }^{69}$. iTOL ${ }^{70}$ was used for

548 the visualization of the phylogenetic tree.

550 Detection of murG genes in Streptomyces and Kitasatospora species

551 MurG domains were predicted using the Pfam database ${ }^{44}$. Proteins with the predicted MurG

552 domains were used to search in the complete protein sets encoded within the extracted

553 genomes using HMMsearch. Instead of a multiple sequence alignment each protein domain 
554 sequence was aligned to its profile Hidden Markov model from Pfam using the HMMalign

555 tool ${ }^{71}$. For each protein a pairwise distance was calculated for all detected MurG proteins

556 and the threshold was set at 0.9. Network visualizations were constructed using Cytoscape

$557 \quad$ (v. 3.7.1) ${ }^{72}$.

558

\section{ACKNOWLEDGEMENTS}

560 We are grateful to Marc Bramkamp for providing us with DivIVA antibodies, and to Eveline

561 Ultee, Joeri Wondergem and Doris Heinrich for help with microscopy. This work was

562 supported by a VIDI grant (12957) from the Dutch Applied Research Council to D.C. and by

563 grant 15812 from NWO-TTW to G.P.v.W. and D.C. 
564 Table 1. Muropeptides identified in $K$. viridifaciens strains grown as mycelium. Monomers

565 and dimers are treated as separate sets. Masses are indicated in Da.

\begin{tabular}{|c|c|c|c|c|c|c|c|}
\hline Peak & Muropeptide & $\begin{array}{c}\text { Retention time } \\
(\min )\end{array}$ & $\begin{array}{c}\text { Observed } \\
\text { Mass }[\mathrm{M}+\mathrm{H}]\end{array}$ & $\begin{array}{c}\text { Calculated } \\
\text { Mass }\end{array}$ & WT & alpha & $\Delta d c w+d i v I V A$ \\
\hline 1 & Tri (-Gly) & 3.46 & 870.39 & 869.38 & $0.69 \%$ & $1.95 \%$ & $0.48 \%$ \\
\hline 2 & Di [deAc] & 3.54 & 656.30 & 655.29 & $0.48 \%$ & $0.10 \%$ & $0.59 \%$ \\
\hline 3 & $\mathrm{Di}$ & 4.07 & 698.31 & 697.30 & $9.39 \%$ & $10.74 \%$ & $6.55 \%$ \\
\hline 4 & Tri & 4.07 & 927.41 & 926.41 & $15.76 \%$ & $22.06 \%$ & $17.34 \%$ \\
\hline 5 & Tetra [Gly4] & 4.13 & 984.44 & 983.43 & $3.03 \%$ & $5.16 \%$ & $5.45 \%$ \\
\hline 6 & TriTri (-GM) & 4.23 & 1355.61 & 1354.60 & $1.16 \%$ & $1.67 \%$ & $0.47 \%$ \\
\hline 7 & Tetra (-Gly) & 4.27 & 941.43 & 940.42 & $1.00 \%$ & $1.71 \%$ & $0.67 \%$ \\
\hline 8 & Tri [Glu] & 4.34 & 928.40 & 927.39 & $1.59 \%$ & $0.42 \%$ & $1.57 \%$ \\
\hline 9 & Penta [Gly5] & 4.38 & 1055.47 & 1054.47 & $21.87 \%$ & $4.02 \%$ & $2.98 \%$ \\
\hline 10 & TetraTetra (-GM) [Gly4] & 4.52 & 1483.67 & 1462.66 & $1.32 \%$ & $2.47 \%$ & $3.45 \%$ \\
\hline 11 & Tetra & 4.58 & 998.45 & 997.44 & $26.66 \%$ & $27.63 \%$ & $25.82 \%$ \\
\hline 12 & TetraTri (-GM) & 4.66 & 1426.65 & 1425.64 & $14.12 \%$ & $18.68 \%$ & $19.13 \%$ \\
\hline 13 & Unidentified peptide & 4.75 & 1055.50 & 1054.47 & $0.00 \%$ & $0.00 \%$ & $5.76 \%$ \\
\hline 14 & Penta & 4.81 & 1069.49 & 1068.48 & $17.49 \%$ & $21.81 \%$ & $29.76 \%$ \\
\hline 15 & TetraTri (-GM) [deAc/Gly4] & 5.01 & 1369.63 & 1368.62 & $6.09 \%$ & $5.96 \%$ & $5.99 \%$ \\
\hline 16 & TetraTetra (-GM) & 5.06 & 1497.39 & 1496.38 & $6.41 \%$ & $6.35 \%$ & $9.82 \%$ \\
\hline 17 & Penta [Glu] & 5.17 & 1070.47 & 1069.47 & $2.05 \%$ & $4.40 \%$ & $3.03 \%$ \\
\hline 18 & TriTri & 5.52 & 1835.81 & 1834.81 & $5.12 \%$ & $5.59 \%$ & $3.75 \%$ \\
\hline 19 & TetraTri [Glu] & 6.11 & 1906.84 & 1905.84 & $4.60 \%$ & $7.42 \%$ & $2.59 \%$ \\
\hline 20 & TetraTri & 6.34 & 1907.83 & 1906.83 & $24.69 \%$ & $20.24 \%$ & $17.17 \%$ \\
\hline 21 & TetraTetra [Glu] & 6.45 & 1977.87 & 1976.88 & $3.97 \%$ & $5.19 \%$ & $7.51 \%$ \\
\hline 22 & TetraTetra & 6.67 & 1978.88 & 1977.86 & $20.50 \%$ & $15.85 \%$ & $15.20 \%$ \\
\hline 23 & PentaTetra [Glu] & 6.94 & 2049.91 & 2048.90 & $12.03 \%$ & $10.57 \%$ & $14.93 \%$ \\
\hline
\end{tabular}

566 


\section{FIGURE LEGENDS}

569 Figure 1. Morphological transitions of the shape-shifting strain alpha. (A) Growth of

570 the $K$. viridifaciens alpha strain on LPMA medium yields green, mucoid colonies exclusively

571 consisting of L-form cells, unlike the wild-type strain that forms yellowish colonies consisting

572 of mycelia and S-cells (B). (C) Time-lapse microscopy stills of alpha proliferating in the wall-

573 deficient state in liquid LPB medium. The arrowhead shows the mother cell, which generates

574 progeny and lyses after $580 \mathrm{~min}$ (marked with an asterisk). Stills were taken from

575 Supplementary Movie 1. (D) Transmission electron microscopy of a wall-deficient cell of

576 alpha. (E) Growth of alpha on solid MYM medium yields compact, non-sporulating colonies

577 unlike the wild-type strain that forms grey-pigmented sporulating colonies (F). (G) Time-

578 lapse microscopy stills of mycelium of alpha transferred to LPMA medium, which show the

579 extrusion of L-forms by filaments (see arrowheads). Stills were taken from Supplementary

580 Movie 2. Scale bars represents $20 \mu \mathrm{m}$ (A, B), $10 \mu \mathrm{m}$ (C, E, F) and $500 \mathrm{~nm}$ (D).

582 Figure 2. The absence of DivIVA abolishes switching of alpha from the wall-deficient

583 to the filamentous mode-of-growth. (A) Growth curves of alpha (black spheres), the

584 divIVA mutant (grey squares) and the dcw mutant (grey triangles) in liquid LPB medium. (B)

585 While all strains grow on LPMA medium, those lacking divIVA are unable to switch to the

586 mycelial mode-of-growth on MYM medium lacking osmoprotectants. (C) Western Blot

587 analysis using antibodies against the C. glutamicum DivIVA protein confirm the absence of

588 DivIVA in the constructed $\triangle \operatorname{div} I V A$ and dcw mutants. Reintroduction of $\operatorname{div} I V A$ under control

589 of the gap1 promoter restores the expression of DivIVA in the divIVA mutant and the ability

590 to form mycelial colonies (see panel B). (D) Comparative LC-MS analysis of peptidoglycan

591 precursors in alpha and its divIVA and dcw mutants. Like the wild-type, all strains produce

592 peptidoglycan precursors including UDP-MurNAc-pentapeptide, which is the last cytosolic 
593 precursor in the PG biosynthesis pathway. (E) MS-MS analysis demonstrating that the

594 product with a mass of 1194.35 is the precursor UDP-MurNAc-pentapeptide.

596 Figure 3. Reintroduction of divIVA alone is sufficient to restore filamentous growth of

597 the dcw mutant. (A) Morphological comparison between alpha (left) and the dcw mutant

598 transformed with $\mathrm{P}_{\text {gap } 1-d i v I V A}$ (right) grown on MYM medium. Unlike alpha, the dcw mutant

599 expressing DivIVA forms colonies with a heterogenous appearance. (B) Peptidoglycan

600 architecture analysis of mycelium of the wild-type strain (top), alpha (middle) and the dcw

601 mutant expressing DivIVA (bottom). The abundance of muropeptides is similar in all strains

602 despite the lack of murG in the $d c w$ mutant (see also Table 1). Scale bar, $40 \mu \mathrm{m}$.

603

604 Figure 4. Overview of MurG and MurG-like proteins present in Streptomyces and

605 Kitasatospora species. The phylogenetic tree was constructed on the basis of four 4 606 conserved housekeeping proteins (AtpD, RecA, TrpB and GyrB). Yellow and purple colors

607 in the inner circle represent Streptomyces and Kitasatospora species, respectively. Strains

608 present in the $\mathrm{NCBI}$ database are indicated in grey in the middle circle, while those from an

609 in-house collection are indicated in red. The pink triangles represent MurG proteins encoded

610 in the dcw gene cluster. The green dots represent distant MurG proteins, whose genes are

611 located elsewhere in the genomes. Phylogenetic trees were constructed using iTOL ${ }^{70}$.

613 Figure 5. MurG2 can functionally replace MurG in peptidoglycan synthesis. (A) Plates

614 of alpha and the $\Delta$ murG, $\Delta$ murG2 and the merodiploid $\triangle$ murG $\Delta$ murG2 strains on LPMA

615 medium (top). With the exception of the $\Delta$ murG $\Delta$ murG2 merodiploid, all strains efficiently

616 switched to filamentous growth on MYM medium lacking osmolytes (bottom). (B) Plates of

$617 K$. viridifaciens and its $\Delta$ murG and $\Delta$ murG2 mutants grown on MYM medium for 7 days. (C)

618 Plates of $K$. viridifaciens and the $\Delta m u r G$ and $\Delta m u r G 2$ mutant strains grown on MYM medium 
619 for 2 (left) or 5 (right) days in the presence of ampicillin (top), penicillin (middle) and

620 tetracycline (bottom). The antibiotic concentrations (in $\mu \mathrm{g} \mathrm{ml}^{-1}$ ) are indicated above the 621 plates.

623 Figure 6. Ectopic expression of murG2 allows silencing of murGsc via CRISPRi.

624 CRISPRi constructs were introduced into S. coelicolor M145 or with control plasmid pMS82

625 and a recombinant strain with pGWS1372 integrated in its genome, thus expressing $K$.

626 viridifaciens MurG2. Expectedly, no effect was seen when CRISPRi constructs were

627 introduced that either had no spacer or that contained a spacer targeting the template strand

628 (T) of murGsc. However, constructs targeting the non-template strand (NT) resulted in

629 severe phenotypic defects and sick colonies of $S$. coelicolor that lacked murG2, but not in 630 pGWS1379 transformants that expressed murG2. Images were taken after 5 days 631 incubation at $30^{\circ} \mathrm{C}$. Bar, $2 \mathrm{~mm}$.

633 Supplementary Figure 1. Comparative analysis of dcw gene clusters from different 634 bacteria. (A) Organization and content of the dcw gene cluster from Streptomyces coelicolor 635 A3(2). (B) MultiGeneBlast output showing homologous dcw gene clusters with a minimal 636 identity of $30 \%$ and minimal sequence coverage of $25 \%$ to the S. coelicolor cluster.

638 Supplementary Figure 2. Localization of DivIVA-eGFP in alpha. (A) Fluorescence 639 microscopy analysis of alpha grown in TSBS medium as a mycelium and carrying pKR1 (left 640 panels), pGreen (middle panels) or pKR2 (right panels). In mycelium containing pKR2, 641 localization of DivIVA-eGFP is found at the hyphal tips (see arrowheads in right panels). No 642 fluorescence is observed in mycelium containing the control plasmid pKR1 (left panels), 643 while a cytosolic signal is observed in alpha transformed with pGreen (middle panels). (B) 644 Fluorescence microscopy analaysis of alpha grown in LPB medium in the wall-deficient state 
645 and carrying pKR1 (left panels), pGreen (middle panels) and pKR2 (right panels). Cells

646 expressing the DivIVA-eGFP fusion protein show distinct foci localized to the membrane

647 (right panels). Like in mycelia, no fluorescence is observed in cells containing the control

648 plasmid pKR1 (left panels), while a cytosolic signal is evident in cells containing pGreen

649 (middle panels). Scale bars represent $10 \mu \mathrm{m}$.

651 Supplementary Figure 3. PCR verification demonstrating the deletions of divIVA and

652 the partial dcw gene cluster in alpha. (A) Schematic illustration of the dcw clusters in 653 alpha (top) and the derivative strains lacking divIVA (middle) or part of the dcw cluster 654 (bottom). To verify the deletions, PCR analyses were performed using primers divIVA-FW 655 and $\operatorname{divIVA}-R v(\mathrm{~B})$ and $d c w-F w$ and $d c w-R v(\mathrm{C})$. (B) PCR analysis using primers divIVA-Fw 656 and divIVA-Rv yielded PCR products of $1.8 \mathrm{~Kb}$ when chromosomal DNA of the wild-type 657 strain (DSM40239) or alpha were used, while a $2.7 \mathrm{~Kb}$ fragment was obtained in the $\triangle$ divIVA 658 mutant. As expected, no product was obtained with these primers using chromosomal DNA 659 of the dcw mutant as the template. (C) PCR analysis using primers $d c w-F w$ and $d c w-R v$ only 660 yielded a PCR product of $1.7 \mathrm{~Kb}$ when chromosomal DNA of the dcw mutant was used as 661 the template. Please note that the sizes of the fragments expected for the wild-type strain 662 and alpha $(8.2 \mathrm{~Kb})$ and the $\triangle$ divIVA mutant $(9.2 \mathrm{~Kb})$ are too large for efficient amplification.

664 Supplementary Figure 4. Domain structure of MurG and MurG2 proteins. MurG 665 proteins contain an N-terminal domain (PF03033) that binds Lipid I and is involved in 666 membrane association. The C-terminal domain (PF04101) contains the UDP-GIcNAc 667 binding site. These domains are found in MurG proteins of E. coli (AAC73201.1), B. subtilis 668 (CAB13395.2), S. coelicolor (NP_626343.1) and K. viridifaciens (BOQ63_RS32465). 669 Notably, MurG2 of K. viridifaciens (BOQ63_RS12640) also contains both domains. Please 
670 note that the protein encoded by the BOQ63_RS05415 gene only contains the N-terminal

671 domain (PF03033), but not the C-terminal (PF04101) domain.

672

673 Supplementary Figure 5. Sequence similarity network of the MurG and MurG2

674 proteins encoded in the genomes of Streptomyces and Kitasatospora species. Nodes

675 represent MurG proteins and edges highlight similarity (with a threshold set at 0.9 ). Node

676 colors indicate if the MurG(-like) proteins are encoded in the dcw gene cluster (red) or

677 elsewhere in the genome (green). Circular node shapes are proteins from Streptomyces

678 spp., while those from Kitasatospora spp. are shown as diamonds. Please note that almost

679 all MurG proteins encoded in the dcw cluster group together.

680

681 Supplementary Figure 6. PCR analysis demonstrating the murG and murG2 deletions

682 in alpha. The deletion of murG and murG2 in alpha was verified by PCR. In strains carrying

683 a wild-type murG gene (DSM40239, alpha and $\Delta$ murG2) a fragment of $1.3 \mathrm{~Kb}$ is amplified.

684 In contrast, a fragment of $1.4 \mathrm{~Kb}$ is found in murG mutants ( $\Delta$ murG and $\Delta m u r G / \Delta m u r G 2$;

685 left gel). Likewise, the expected PCR product for strains carrying the murG2 wild-type gene

686 (DSM40239, alpha, $\Delta$ murG) was $1.2 \mathrm{~Kb}$, while replacement of murG2 by apramycin or

687 viomycin yielded PCR products of $1.3 \mathrm{~Kb}$ and $1.5 \mathrm{~Kb}$, respectively (right gel). Please note

688 that the murG2 gene is still detectable in the $\Delta$ murG $\Delta$ murG2 merodiploid.

689

690 Supplementary Figure 7. PCR analysis demonstrating the murG and murG2 deletions

691 in Kitasatospora viridifaciens. The deletion of murG and murG2 in K. viridifaciens was

692 verified by PCR. In the wild-type strain (DSM40239) a fragment of 1365 bp is amplified,

693 while a fragment of $1436 \mathrm{bp}$ is found in three independent murG mutants ( $\Delta$ murG; left gel).

694 Likewise, the expected size of the PCR product for the wild-type strain carrying the murG2 
695 gene (DSM40239) was 1279 bp, while replacement of murG2 yielded a PCR product of

6961311 bp (AmurG2; right gel).

698 Supplementary Movie 1. L-form proliferation of alpha. Time-lapse microscopy showing

699 proliferation of alpha in LPB medium containing high levels of sucrose. The times are

700 indicated in $\min$. The scale bar indicates $5 \mu \mathrm{m}$.

701

702 Supplementary Movie 2. Extrusion of L-forms from hyphal tips. Cell wall-deficient L-

703 forms are extruded from hyphal tips when mycelium of alpha is transferred to LPMA agar

704 containing high levels of sucrose. The times are indicated in $\min$. The scale bar indicates 5

$705 \mu \mathrm{m}$.

706

\section{REFERENCES}

7081 Liu, Y. \& Breukink, E. The membrane steps of bacterial cell wall synthesis as antibiotic targets. Antibiotics (Basel) 5, doi:10.3390/antibiotics5030028 (2016).

711

712

713

714

715

716

717

718 2 Mohammadi, T. et al. Identification of FtsW as a transporter of lipid-linked cell wall

3 Sham, L. T. et al. MurJ is the flippase of lipid-linked precursors for peptidoglycan biogenesis. Science 345, 220-222, doi:10.1126/science.1254522 (2014).

4 Meeske, A. J. et al. MurJ and a novel lipid II flippase are required for cell wall biogenesis in Bacillus subtilis. Proc Natl Acad Sci U S A 112, 6437-6442, doi:10.1073/pnas.1504967112 (2015).

5 Scheffers, D. J. \& Pinho, M. G. Bacterial cell wall synthesis: new insights from localization studies. Microbiol Mol Biol Rev 69, 585-607 (2005).

6 Meeske, A. J. et al. SEDS proteins are a widespread family of bacterial cell wall polymerases. Nature 537, 634-638, doi:10.1038/nature19331 (2016).

7 Cho, $\mathrm{H}$. et al. Bacterial cell wall biogenesis is mediated by SEDS and PBP polymerase families functioning semi-autonomously. Nat Microbiol 1, 16172, doi:10.1038/nmicrobiol.2016.172 (2016).

8 Pazos, M., Peters, K. \& Vollmer, W. Robust peptidoglycan growth by dynamic and variable multi-protein complexes. Curr Opin Microbiol 36, 55-61, doi:10.1016/j.mib.2017.01.006 (2017).

9 Vicente, M. \& Errington, J. Structure, function and controls in microbial division. Mol Microbiol 20, 1-7 (1996).

10 Tamames, J., González-Moreno, M., Mingorance, J., Valencia, A. \& Vicente, M. Bringing gene order into bacterial shape. Trends Genet 17, 124-126 (2001). division. J Mol Recognit 17, 481-487, doi:10.1002/jmr.718 (2004). 
73312 Claessen, D., Rozen, D. E., Kuipers, O. P., Søgaard-Andersen, L. \& van Wezel, G. $P$. Bacterial solutions to multicellularity: a tale of biofilms, filaments and fruiting bodies. Nat Rev Microbiol 12, 115-124, doi:10.1038/nrmicro3178 (2014).

13 Flärdh, K. \& Buttner, M. J. Streptomyces morphogenetics: dissecting differentiation in a filamentous bacterium. Nat Rev Microbiol 7, 36-49, doi:10.1038/nrmicro1968 (2009).

738

14 Barka, E. A. et al. Taxonomy, physiology, and natural products of Actinobacteria. Microbiol Mol Biol Rev 80, 1-43, doi:10.1128/MMBR.00019-15 (2016).

741

15 Bérdy, J. Thoughts and facts about antibiotics: where we are now and where we are heading. J Antibiot (Tokyo) 65, 385-395, doi:10.1038/ja.2012.27 (2012).

742

743

16 Celler, K., Koning, R. I., Willemse, J., Koster, A. J. \& van Wezel, G. P. Crossmembranes orchestrate compartmentalization and morphogenesis in Streptomyces.

744

745 Nat Commun 7, ncomms11836, doi:10.1038/ncomms11836 (2016).

17 Wildermuth, H. \& Hopwood, D. A. Septation during sporulation in Streptomyces coelicolor. J Gen Microbiol 60, 51-59 (1970).

18 Manteca, A., Fernandez, M. \& Sanchez, J. A death round affecting a young compartmentalized mycelium precedes aerial mycelium dismantling in confluent surface cultures of Streptomyces antibioticus. Microbiology 151, 3689-3697, doi:10.1099/mic.0.28045-0 (2005).

19 Tenconi, E., Traxler, M. F., Hoebreck, C., van Wezel, G. P. \& Rigali, S. Production of prodiginines is part of a programmed cell death process in Streptomyces coelicolor. Front Microbio/ 9, 1742, doi:10.3389/fmicb.2018.01742 (2018). Schwedock, J., McCormick, J. R., Angert, E. R., Nodwell, J. R. \& Losick, R. Assembly of the cell division protein FtsZ into ladder-like structures in the aerial hyphae of Streptomyces coelicolor. Mol Microbiol 25, 847-858 (1997).

21 Jakimowicz, D. \& van Wezel, G. P. Cell division and DNA segregation in Streptomyces: how to build a septum in the middle of nowhere? Mol Microbiol 85, 393-404, doi:10.1111/j.1365-2958.2012.08107.x (2012).

761

762

763

764

765

766

767

768

769

770

771

772

773

774

775

776

777

778

779

780

22 McCormick, J. R. \& Losick, R. Cell division gene ftsQ is required for efficient sporulation but not growth and viability in Streptomyces coelicolor A3(2). J Bacteriol 178, 5295-5301 (1996).

23 Edwards, D. H. \& Errington, J. The Bacillus subtilis DivIVA protein targets to the division septum and controls the site specificity of cell division. Mol Microbiol 24, 905915 (1997).

24 Flärdh, K. Essential role of DivIVA in polar growth and morphogenesis in Streptomyces coelicolor A3(2). Mol Microbiol 49, 1523-1536 (2003).

25 McCormick, J. R. Cell division is dispensable but not irrelevant in Streptomyces. Curr Opin Microbiol 12, 689-698, doi:10.1016/j.mib.2009.10.004 (2009).

26 Bagchi, S., Tomenius, H., Belova, L. M. \& Ausmees, N. Intermediate filament-like proteins in bacteria and a cytoskeletal function in Streptomyces. Mol Microbiol 70, 1037-1050, doi:10.1111/j.1365-2958.2008.06473.x (2008).

27 Celler, K., Koning, R. I., Koster, A. J. \& van Wezel, G. P. Multidimensional view of the bacterial cytoskeleton. J Bacteriol 195, 1627-1636, doi:10.1128/JB.02194-12 (2013).

28 Holmes, N. A. et al. Coiled-coil protein Scy is a key component of a multiprotein assembly controlling polarized growth in Streptomyces. Proc Natl Acad Sci U S A 110, E397-406, doi:10.1073/pnas.1210657110 (2013).

29 Fuchino, K. et al. Dynamic gradients of an intermediate filament-like cytoskeleton are recruited by a polarity landmark during apical growth. Proc Natl Acad Sci U S A 110, E1889-1897, doi:10.1073/pnas.1305358110 (2013). 
78330 Girard, G. et al. A novel taxonomic marker that discriminates between

784

785

786 morphologically complex actinomycetes. Open Biol 3, 130073, doi:10.1098/rsob.130073 (2013).

787

31 Girard, G. et al. Analysis of novel kitasatosporae reveals significant evolutionary changes in conserved developmental genes between Kitasatospora and Streptomyces. Antonie Van Leeuwenhoek 106, 365-380, doi:10.1007/s10482-0140209-1 (2014).

789

790

32 Ramijan, K. et al. Stress-induced formation of cell wall-deficient cells in filamentous

791

792 actinomycetes. Nat Commun 9, 5164, doi:https://doi.org/10.1101/094037 (2018).

793

794

795

796

797

33 Claessen, D. \& Errington, J. Cell wall-deficiency as a coping strategy for stress. Trends in Microbiology (2019).

34 Errington, J., Mickiewicz, K., Kawai, Y. \& Wu, L. J. L-form bacteria, chronic diseases and the origins of life. Phil Trans $R$ Soc $B$ 371, 20150494, doi:10.1098/rstb.2015.0494 (2016).

35 Leaver, M., Dominguez-Cuevas, P., Coxhead, J. M., Daniel, R. A. \& Errington, J. Life without a wall or division machine in Bacillus subtilis. Nature 457, 849-853, doi:10.1038/nature07742 (2009).

799

800

36 Mercier, R., Kawai, Y. \& Errington, J. Wall proficient E. coli capable of sustained growth in the absence of the Z-ring division machine. Nature Microbiology 1, 16091, doi:10.1038/nmicrobiol.2016.91 (2016).

803

37 Studer, P. et al. Proliferation of Listeria monocytogenes L-form cells by formation of internal and external vesicles. Nat Commun 7, 13631, doi:10.1038/ncomms13631 (2016).

805

806

38 Mercier, R., Kawai, Y. \& Errington, J. Excess membrane synthesis drives a primitive

807

808 mode of cell proliferation. Cell 152, 997-1007, doi:10.1016/j.cell.2013.01.043 (2013).

39 Errington, J. L-form bacteria, cell walls and the origins of life. Open Biol 3, 120143, doi:10.1098/rsob.120143 (2013).

810

40 Briers, Y., Walde, P., Schuppler, M. \& Loessner, M. J. How did bacterial ancestors reproduce? Lessons from L-form cells and giant lipid vesicles: multiplication similarities between lipid vesicles and L-form bacteria. Bioessays 34, 1078-1084, doi:10.1002/bies.201200080 (2012).

813

814

41 Cambré, A. et al. Metabolite profiling and peptidoglycan analysis of transient cell wall-deficient bacteria in a new Escherichia coli model system. Environ Microbiol, doi:10.1111/1462-2920.12594 (2014).

816

817

42 Mercier, R., Kawai, Y. \& Errington, J. General principles for the formation and proliferation of a wall-free (L-form) state in bacteria. Elife 3, 04629, doi:10.7554/eLife.04629 (2014).

819

820

821

822

823

824

825

826

827

828

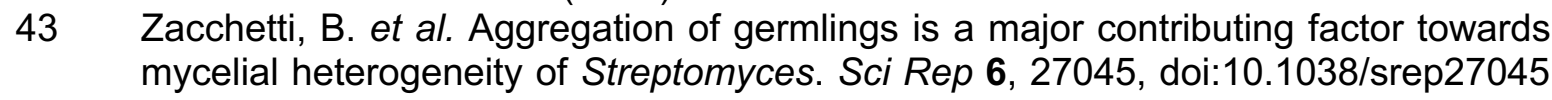
(2016).

44 El-Gebali, S. et al. The Pfam protein families database in 2019. Nucleic Acids Res 47, D427-D432, doi:10.1093/nar/gky995 (2019).

45 Qi, L. S. et al. Repurposing CRISPR as an RNA-guided platform for sequencespecific control of gene expression. Cell 152, 1173-1183, doi:10.1016/j.cell.2013.02.022 (2013).

46 Ultee, E. et al. Teichoic acids anchor distinct cell wall lamellae in an apically growing bacterium. Commun Biol 3, 314, doi:10.1101/714758 (2020). 254 (1994). 
83348 Bennett, J. A., Aimino, R. M. \& McCormick, J. R. Streptomyces coelicolor genes ftsL and divIC play a role in cell division but are dispensable for colony formation. $J$

835

836 Bacteriol 189, 8982-8992, doi:10.1128/JB.01303-07 (2007).

837

49 Bennett, J. A. et al. Medium-dependent phenotypes of Streptomyces coelicolor with mutations in ftsl or ftsW. J Bacteriol 191, 661-664, doi:10.1128/JB.01048-08 (2009).

838

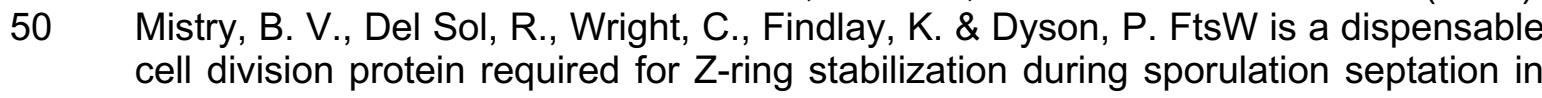

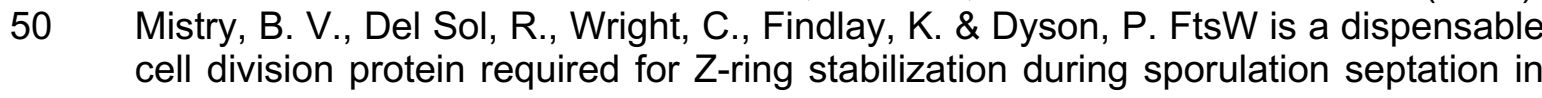

839

840

841

842 Streptomyces coelicolor. J Bacteriol 190, 5555-5566, doi:10.1128/JB.00398-08 (2008).

843

844

845

51 Labeda, D. P. et al. Phylogenetic study of the species within the family Streptomycetaceae. Antonie van Leeuwenhoek 101, 73-104, doi:10.1007/s10482011-9656-0 (2012).

846

847

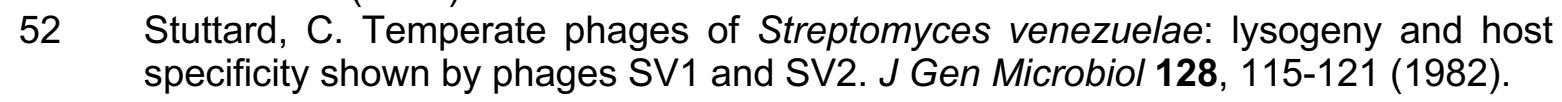

53 Sun, J., Kelemen, G. H., Fernández-Abalos, J. M. \& Bibb, M. J. Green fluorescent protein as a reporter for spatial and temporal gene expression in Streptomyces coelicolor A3(2). Microbiology 145, 2221-2227 (1999).

54 Gust, B., Challis, G. L., Fowler, K., Kieser, T. \& Chater, K. F. PCR-targeted Streptomyces gene replacement identifies a protein domain needed for biosynthesis of the sesquiterpene soil odor geosmin. Proc Natl Acad Sci U S A 100, 1541-1546, doi:10.1073/pnas.0337542100 (2003).

854

55 Ramijan, K., van Wezel, G. P. \& Claessen, D. Genome sequence of the filamentous actinomycete Kitasatospora viridifaciens. Genome Announc 5, e01560-01516, doi:10.1128/genomeA.01560-16 (2017).

856

857

56 Vara, J., Lewandowska-Skarbek, M., Wang, Y. G., Donadio, S. \& Hutchinson, C. R. Cloning of genes governing the deoxysugar portion of the erythromycin biosynthesis pathway in Saccharopolyspora erythraea (Streptomyces erythreus). J Bacteriol 171, 5872-5881 (1989).

861

862

863

864

865

866

867

868

869

870

871

872

873

874

875

876

877

878

879

880

881

57 Światek, M. A., Tenconi, E., Rigali, S. \& van Wezel, G. P. Functional analysis of the $\mathrm{N}$-acetylglucosamine metabolic genes of Streptomyces coelicolor and role in control of development and antibiotic production. $J$ Bacteriol 194, 1136-1144, doi:10.1128/JB.06370-11 (2012).

58 Motamedi, H., Shafiee, A. \& Cai, S.-J. Integrative vectors for heterologous gene expression in Streptomyces spp. Gene 160, 25-31 (1995).

59 Gregory, M. A., Till, R. \& Smith, M. C. M. Integration site for Streptomyces phage phiBT1 and development of site-specific integrating vectors. J Bacteriol 185, 53205323 (2003).

60 Kieser, T., Bibb, M. J., Buttner, M. J., Chater, K. F. \& Hopwood, D. A. Practical Streptomyces genetics. (The John Innes Foundation, 2000).

61 van der Aart, L. T., Lemmens, N., van Wamel, W. J. \& van Wezel, G. P. Substrate inhibition of VanA by d-alanine reduces vancomycin resistance in a VanX-dependent manner. Antimicrob Agents Chemother 60, 4930-4939, doi:10.1128/AAC.00276-16 (2016).

62 van der Aart, L. T. et al. High-resolution analysis of the peptidoglycan composition in Streptomyces coelicolor. J Bacteriol 200, doi:10.1128/JB.00290-18 (2018).

63 Kühner, D., Stahl, M., Demircioglu, D. D. \& Bertsche, U. From cells to muropeptide structures in $24 \mathrm{~h}$ : peptidoglycan mapping by UPLC-MS. Sci Rep 4, 7494, doi:10.1038/srep07494 (2014).

882

883

64 Medema, M. H., Takano, E. \& Breitling, R. Detecting sequence homology at the gene cluster level with MultiGeneBlast. Mol Biol Evol 30, 1218-1223, doi:10.1093/molbev/mst025 (2013). 
88465 Zhu, H. et al. Eliciting antibiotics active against the ESKAPE pathogens in a collection 885 of actinomycetes isolated from mountain soils. Microbiology 160, 1714-1725, 886 doi:10.1099/mic.0.078295-0 (2014).

$88766 \quad$ Finn, R. D., Clements, J. \& Eddy, S. R. HMMER web server: interactive sequence 888 similarity searching. Nucleic Acids Res 39, W29-37, doi:10.1093/nar/gkr367 (2011).

$88967 \quad$ Katoh, K. \& Standley, D. M. MAFFT multiple sequence alignment software version 8907 : improvements in performance and usability. Mol Biol Evol 30, 772-780, 891 doi:10.1093/molbev/mst010 (2013).

89268 Shen, W., Le, S., Li, Y. \& Hu, F. SeqKit: a cross-platform and ultrafast toolkit for 893 FASTA/Q file manipulation. PLoS One 11, e0163962, 894 doi:10.1371/journal.pone.0163962 (2016).

89569 Stamatakis, A. RAxML version 8: a tool for phylogenetic analysis and post-analysis 896 of large phylogenies. Bioinformatics 3 , 897 doi:10.1093/bioinformatics/btu033 (2014).

$89870 \quad$ Letunic, I. \& Bork, P. Interactive Tree Of Life (iTOL) v4: recent updates and new 899 developments. Nucleic Acids Res, doi:10.1093/nar/gkz239 (2019).

90071 Eddy, S. R. Accelerated profile HMM searches. PLoS Comput Biol 7, e1002195, 901 doi:10.1371/journal.pcbi.1002195 (2011).

90272 Shannon, P. et al. Cytoscape: a software environment for integrated models of 903 biomolecular interaction networks. Genome Res 13, 2498-2504, 904 doi:10.1101/gr.1239303 (2003). 

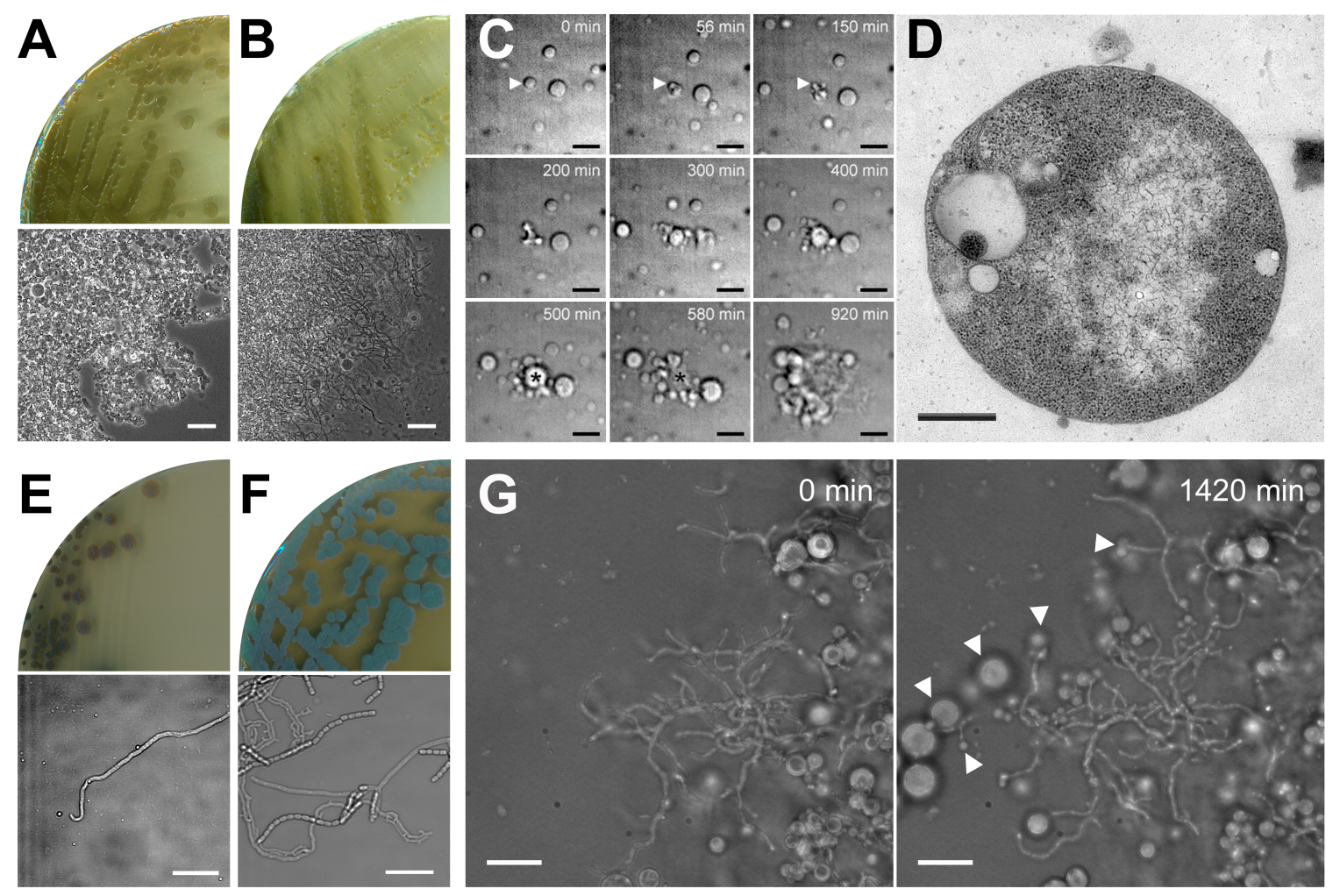

Figure 1. Morphological transitions of the shape-shifting strain alpha. (A) Growth of the $K$. viridifaciens alpha strain on LPMA medium yields green, mucoid colonies exclusively consisting of L-form cells, unlike the wild-type strain that forms yellowish colonies consisting of mycelia and S-cells (B). (C) Time-lapse microscopy stills of alpha proliferating in the walldeficient state in liquid LPB medium. The arrowhead shows the mother cell, which generates progeny and lyses after $580 \mathrm{~min}$ (marked with an asterisk). Stills were taken from Supplementary Movie 1. (D) Transmission electron microscopy of a wall-deficient cell of alpha. (E) Growth of alpha on solid MYM medium yields compact, non-sporulating colonies unlike the wild-type strain that forms grey-pigmented sporulating colonies (F). (G) Timelapse microscopy stills of mycelium of alpha transferred to LPMA medium, which show the extrusion of L-forms by filaments (see arrowheads). Stills were taken from Supplementary Movie 2. Scale bars represents $20 \mu \mathrm{m}(\mathrm{A}, \mathrm{B}), 10 \mu \mathrm{m}(\mathrm{C}, \mathrm{E}, \mathrm{F})$ and $500 \mathrm{~nm}$ (D). 

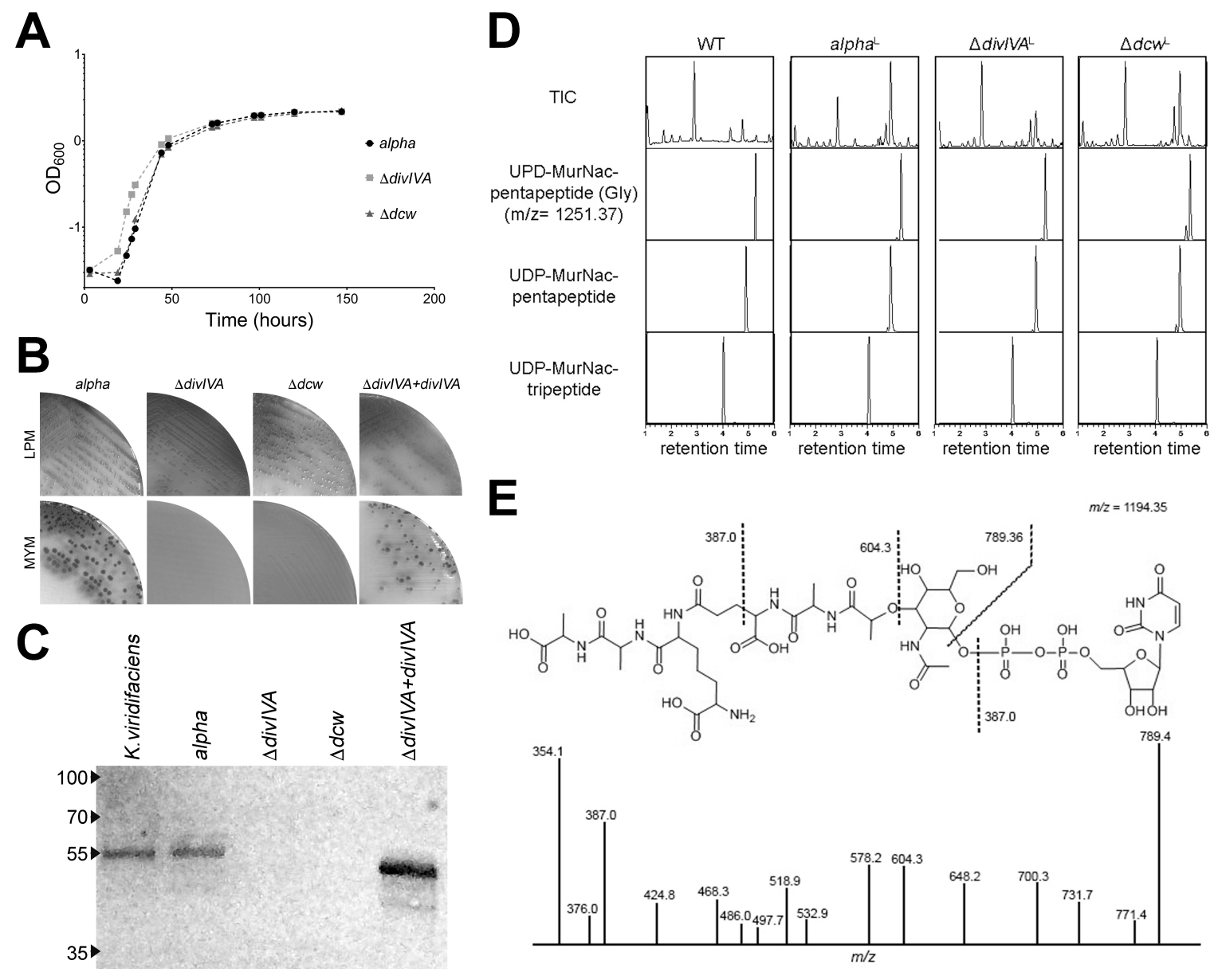

Figure 2. The absence of DivIVA abolishes switching of alpha from the wall-deficient to the filamentous mode-of-growth. (A) Growth curves of alpha (black spheres), the divIVA mutant (grey squares) and the dcw mutant (grey triangles) in liquid LPB medium. (B) While all strains grow on LPMA medium, those lacking divIVA are unable to switch to the mycelial mode-of-growth on MYM medium lacking osmoprotectants. (C) Western Blot analysis using antibodies against the C. glutamicum DivIVA protein confirm the absence of DivIVA in the constructed $\triangle \operatorname{div} I V A$ and $d c w$ mutants. Reintroduction of divIVA under control of the gap1 promoter restores the expression of DivIVA in the divIVA mutant and the ability to form mycelial colonies (see panel B). (D) Comparative LC-MS analysis of peptidoglycan precursors in alpha and its divIVA and dcw mutants. Like the wild-type, all strains produce peptidoglycan precursors including UDP-MurNAc-pentapeptide, which is the last cytosolic precursor in the PG biosynthesis pathway. (E) MS-MS analysis demonstrating that the product with a mass of 1194.35 is the precursor UDP-MurNAc-pentapeptide. 
A
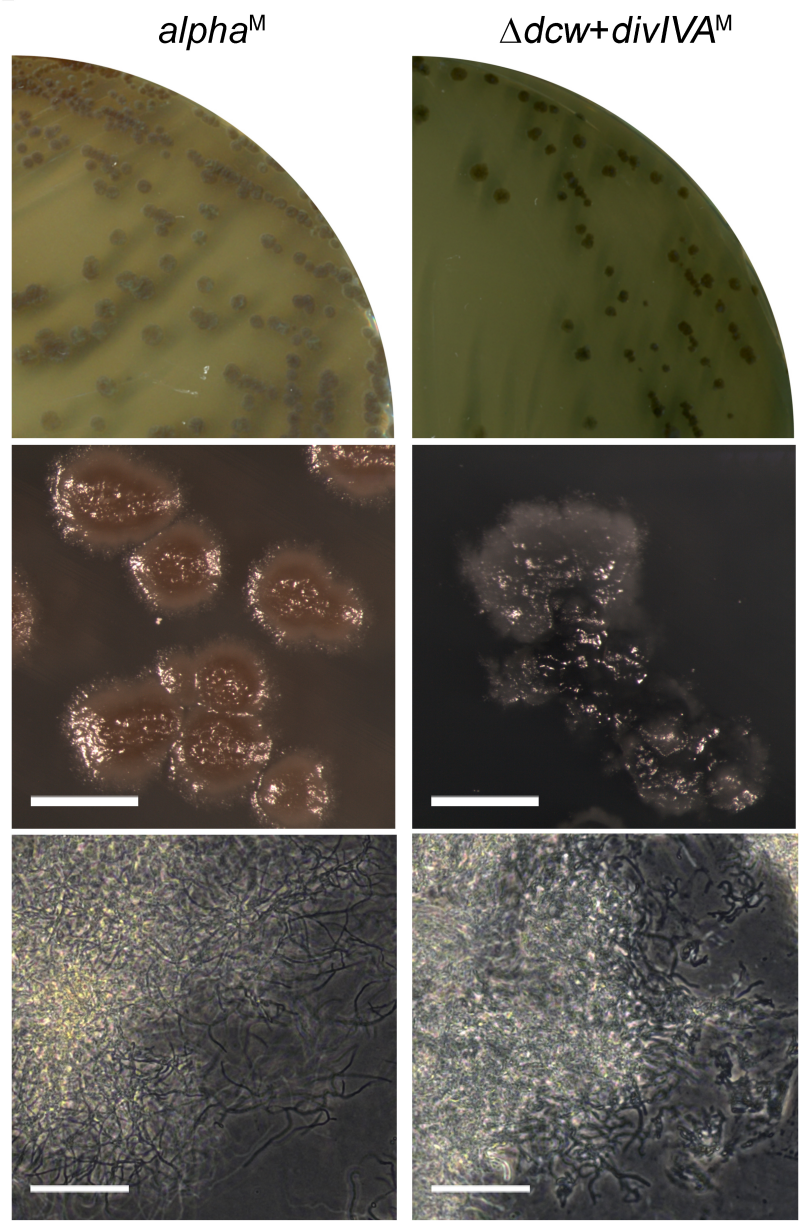

B
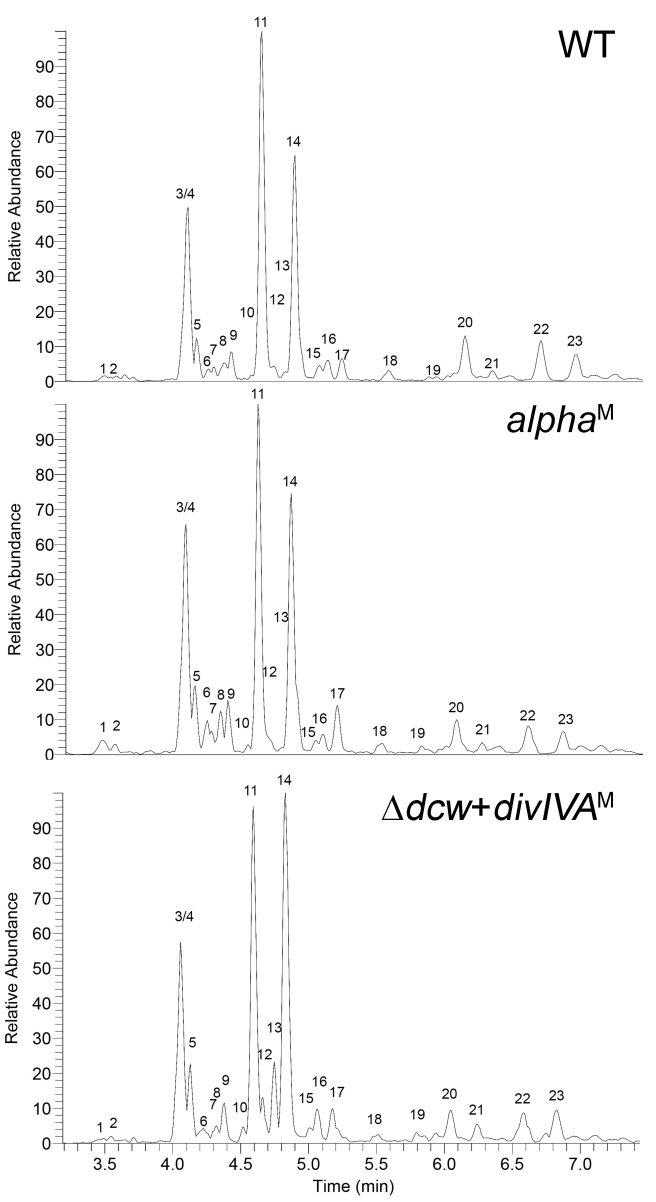

Figure 3. Reintroduction of divIVA alone is sufficient to restore filamentous growth of the dcw mutant. (A) Morphological comparison between alpha (left) and the $d c w$ mutant transformed with $\mathrm{P}_{\text {gap }}$-divIVA (right) grown on MYM medium. Unlike alpha, the dcw mutant expressing DivIVA forms colonies with a heterogenous appearance. (B) Peptidoglycan architecture analysis of mycelium of the wild-type strain (top), alpha (middle) and the $d c w$ mutant expressing DivIVA (bottom). The abundance of muropeptides is similar in all strains despite the lack of murG in the $d c w$ mutant (see also Table 1). Scale bar, $40 \mu \mathrm{m}$. 
bioRxiv preprint doi: https://doi.org/10.1101/2020 10.12336396; this version posted October 12, 2020. The copyright holder for this preprint (which was not certified by peer review) is the author/funder, who has granted bioRxiv a license to display the preprint in perpetuity. It is made available under aCC-BY-NC-ND 4.0 International license.

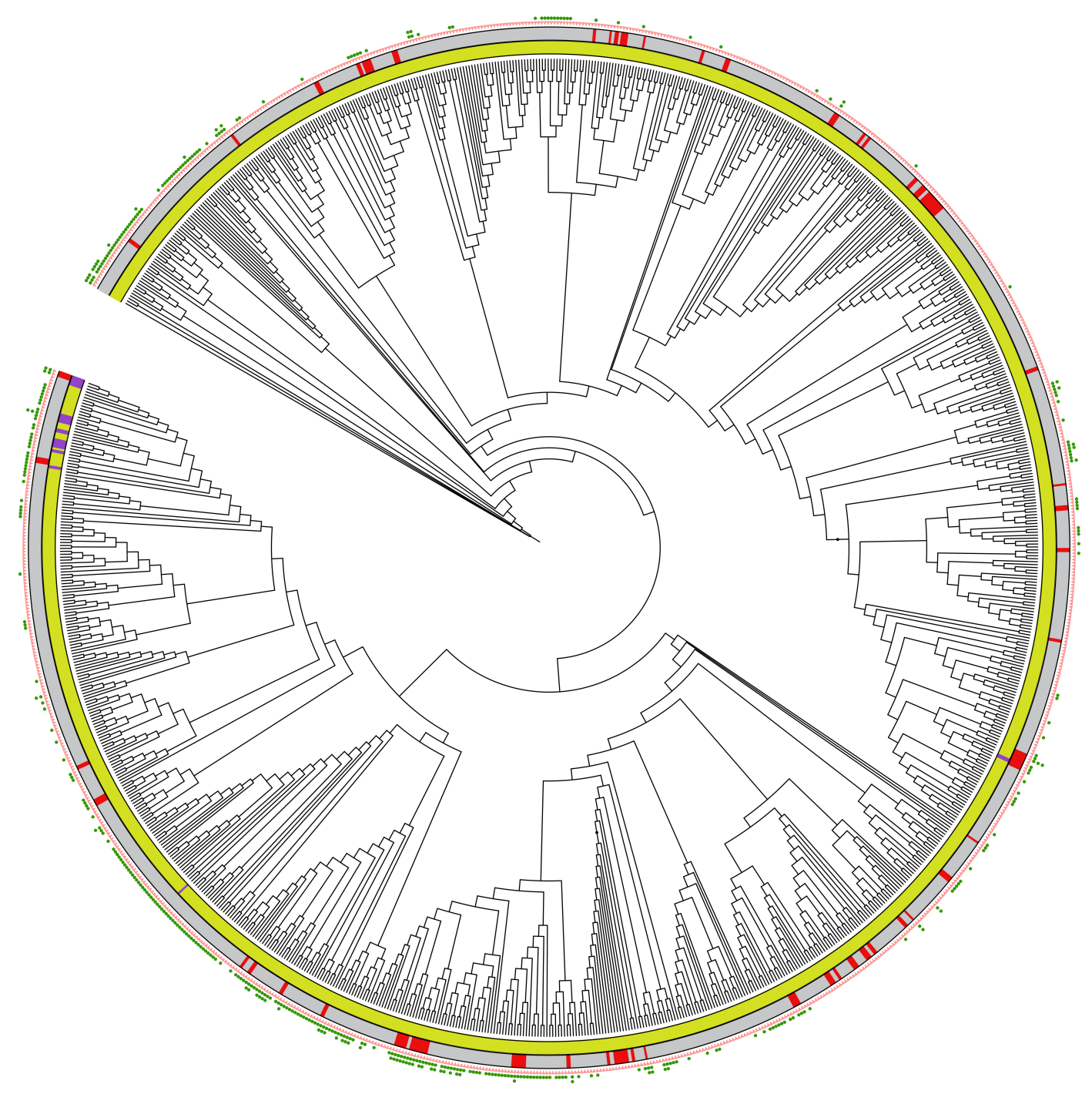

Figure 4. Overview of MurG and MurG-like proteins present in Streptomyces and Kitasatospora species. The phylogenetic tree was constructed on the basis of four 4 conserved housekeeping proteins (AtpD, RecA, TrpB and GyrB). Yellow and purple colors in the inner circle represent Streptomyces and Kitasatospora species, respectively. Strains present in the NCBI database are indicated in grey in the middle circle, while those from an in-house collection are indicated in red. The pink triangles represent MurG proteins encoded in the $d c w$ gene cluster. The green dots represent distant MurG proteins, whose genes are located elsewhere in the genomes. Phylogenetic trees were constructed using iTOL ${ }^{70}$. 


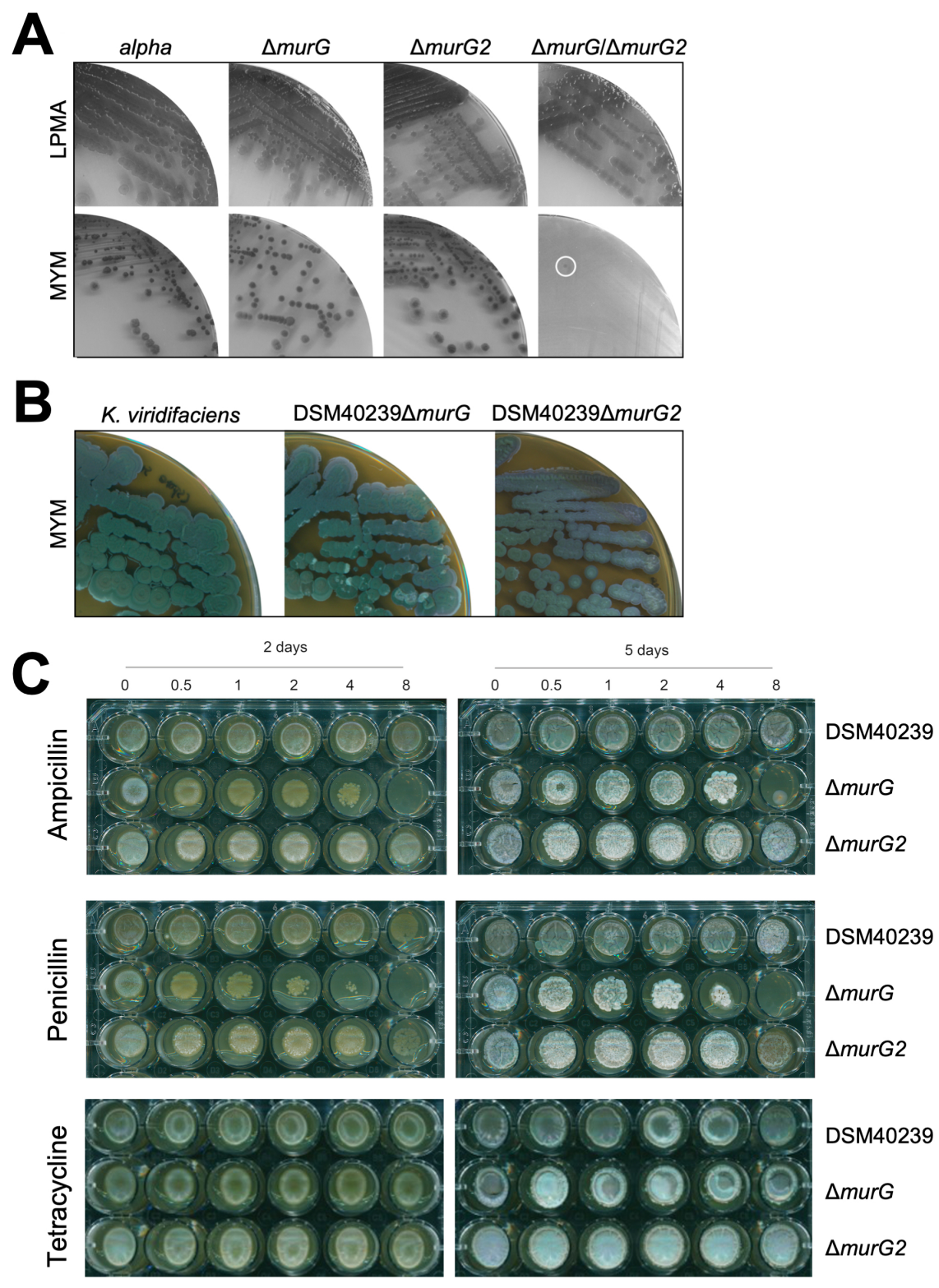

Figure 5. MurG2 can functionally replace MurG in peptidoglycan synthesis. (A) Plates of alpha and the $\Delta$ murG, $\Delta$ murG2 and the merodiploid $\triangle$ murG $\Delta$ murG2 strains on LPMA medium (top). With the exception of the $\Delta$ murG $\Delta$ murG2 merodiploid, all strains efficiently switched to filamentous growth on MYM medium lacking osmolytes (bottom). (B) Plates of $K$. viridifaciens and its $\triangle$ murG and $\triangle$ murG2 mutants grown on MYM medium for 7 days. (C) Plates of $K$. viridifaciens and the $\triangle$ murG and $\triangle$ murG2 mutant strains grown on MYM medium for 2 (left) or 5 (right) days in the presence of ampicillin (top), penicillin (middle) and tetracycline (bottom). The antibiotic concentrations (in $\mu \mathrm{g} \mathrm{ml}^{-1}$ ) are indicated above the plates. 

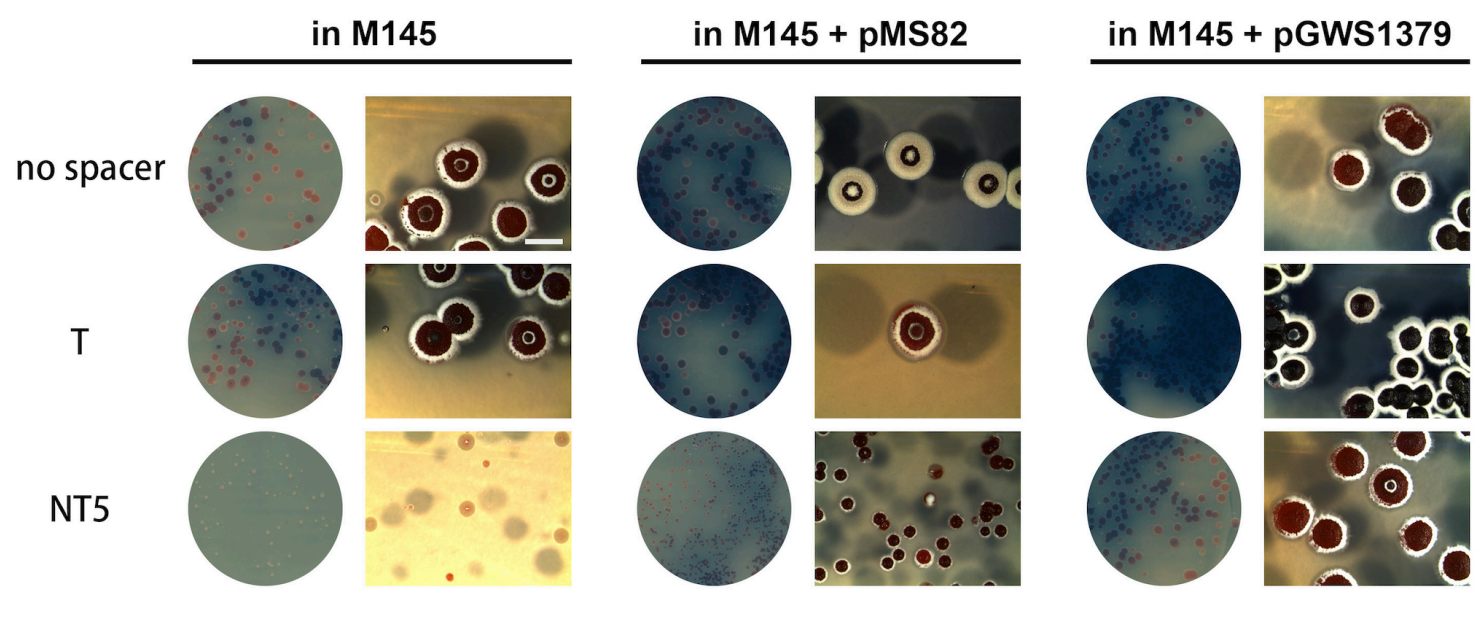

Figure 6. Ectopic expression of murG2 allows silencing of murGsc via CRISPRi. CRISPRi constructs were introduced into $S$. coelicolor M145 or with control plasmid pMS82 and a recombinant strain with pGWS1372 integrated in its genome, thus expressing $K$. viridifaciens MurG2. Expectedly, no effect was seen when CRISPRi constructs were introduced that either had no spacer or that contained a spacer targeting the template strand (T) of murGsc. However, constructs targeting the non-template strand (NT) resulted in severe phenotypic defects and sick colonies of S. coelicolor that lacked murG2, but not in pGWS1379 transformants that expressed murG2. Images were taken after 5 days incubation at $30^{\circ} \mathrm{C}$. Bar, $2 \mathrm{~mm}$. 